pp. 749-764

\title{
A Study on Volume of Difference of Two Joint pdf's, Focused on the Relation to Normal Theory LR Tests
}

\author{
Kwangjin Lee ${ }^{1)}$
}

\begin{abstract}
In this paper we explain that normal theory likelihood-ratio tests $\left(z, t, \chi^{2}, F\right)$ for mean(s) or variance(s) can be geometrically related to volume of difference of two joint pdf's. One is an estimated joint pdf under null parameter space $\omega$ and the other is an estimated joint pdf under full parameter space $\Omega$. For explanations, 'distance between two distributions' is defined. We study properties of it, and derive some results on the distance between two multivariate normal distributions.
\end{abstract}

Keywords : distance between two distributions, $z, t, \chi^{2}, F$-tests, ANOVA, LR-test

\section{1.서론}

정규모집단의 확률표본으로 모평균과 모분산의 가설들에 대한 검정법은 통계적 검정이론 교육 에서 가장 기본이 되는 토픽이다. 이들 중에서도 대표적인 문제들을 정리하면 다음과 같다.

문제 1) 단일표본의 경우 모평균에 관한 검정 - 모분산이 알려진 경우

문제 2) 단일표본의 경우 모평균에 관한 검정 - 모분산이 미지인 경우

문제 3) 단일표본의 경우 모분산에 관한 검정 - 모평균이 미지인 경우

문제 4) 두 독립표본의 경우 모평균의 동일성에 관한 검정 - 모분산들이 알려진 경우

문제 5) 두 독립표본의 경우 모평균의 동일성에 관한 검정 - 등분산이지만 미지인 경우

문제 6) 두 독립표본의 경우 모평균의 동일성에 관한 검정 - 이분산으로 미지인 경우

문제 7) 일원 분산분석

학부 고급 수리통계학의 추정단원에서는 추정량을 구하는 방법으로서 최우추정법, 적률법을 비 롯하여 최소제곱법, 베이즈방법, 최소카이제곱법, 최소거리법 등이 소개-교육되고 있다. 그러나 검정단원에서는 가설검정법을 구하는 방법으로서 거의 독보적인 지위를 차지한다고 할 수 있는 우도비원리(likelihood-ratio principle) 이외에는 특별히 제공되지 않고 있다. 물론 석사과정 수준 이상에서는 합교원리(union-intersection principle)라는 개념이 교육되기도 한다. 사실 우도비라는 개념은 학부 수리통계학 수준에서는 쉽게 교육 또는 이해 할 수 있는 개념이라고 할 수 없다. 이 에 본 연구에서는 최대우도의 비(此)라는 어려운 개념을 사용하지 않고도 귀무가설과 대립가설 각

1 Associate Professor, Department of Information Statistics, Mokwon University, Daejeon, 302-729, Korea,

E-mail : leekj@mokwon.ac.kr 
각의 모수공간상에서 추정된 결합확률밀도함수들의 차의 면적이라는 기하적 개념으로도 문제1) 문제7)의 우도비 검정통계량들이 설명되어질 수 있음을 보이고자 한다.

2절에서는 '분포간 거리'라는 두 확률분포들 사이의 거리를 정의하고 이의 성질들 몇 가지를 살펴본다. 3절에서는 두 다변량정규분포의 확률밀도함수들의 그래프들 사이의 관계를 살펴보고 모 수가 다른 두 다변량정규분포들 사이의 분포간 거리에 대해 탐구한다. 4절에서는 결합분포간 거리 추정량과 우도비 검정통계량과의 관계를 문제 $1,2,3,5,7$ 을 통해 살펴본다.

\section{2.'분포간 거리'의 정의}

[정의1] $n$ 차원의 두 연속형 확률변수벡터 $X_{a}=\left(\begin{array}{lllll}X_{a 1} & X_{a 2} & \cdots & X_{a n}\end{array}\right)^{\prime}$ 와 $X_{b}=\left(\begin{array}{llll}X_{b 1} & X_{b 2} & \cdots & X_{b n}\end{array}\right)^{\prime}$ 의 확률밀도함수를 각각 $f_{a}(x)=f_{a}\left(x_{1}, x_{2}, \cdots, x_{n}\right)$ 와 $f_{b}(x)=f_{b}\left(x_{1}, x_{2}, \cdots, x_{n}\right)$ 라고 했을 때 다음의 $D_{n}(a, b)$ 를 $n$ 차원의 두 확률분포 $f_{a}(x)$ 와 $f_{b}(x)$ 사이의 '분포간 거리'라 정의한다.

$$
D_{n}(a, b)=\frac{1}{2} \int_{R^{n}}\left|f_{a}(x)-f_{b}(x)\right|(d x)
$$

여기서, $R^{n}$ 은 $n$ 차원 유클리드 공간을 의미하며, $(d x)$ 은 $d x_{1} d x_{2} \cdots d x_{n}$ 을 나타낸다.

[그립1]은 $\mathrm{n}=1$ 인 경우에서의 분포간 거리의 기하적 의미를 보여주는 예 그림으로 빗금부분 면적의 반이 두 분포간 거

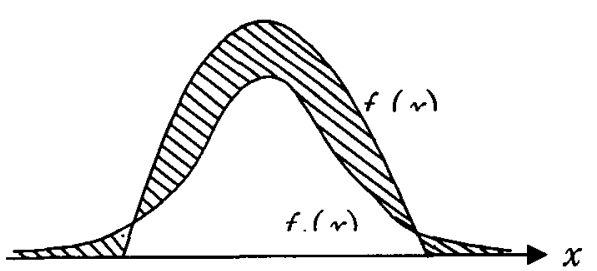

[그림1] $n=1$ 인 경우 두 분포간 거리의 기하적 의미를 보여주는 예 그림 리가 된다. $\mathrm{n}$ 차원의 경우 분포간 거리는 두 확률밀도함수의 초곡면(hyper-surface)들로 둘러싸인 부분의 초부피 (hyper-volume)의 $1 / 2$ 에 해당된다는 기하적 의미를 지닌다. 그리고 이 분포간 거리값이 0 에 가까울수록 두 분포는 '확률 적으로 공유하는 부분이 많음', 1 에 가까울수록 두 분포는 '확률적으로 공유하는 부분이 적음'을 의미한다고 하겠다. 그리고 그 값이 0 이면 두 분포는 '완전 일치', 1 이면 '완전 불 일치'라고 명명할 것이다.

참고로, 분포간 거리 $D_{n}(a, b)$ 는 일반적인 거리조건들 (1) $D_{n}(a, a)=0$, (2) $D_{n}(a, b)=D_{n}(b, a)$, (3) $D_{n}(a, b)+D_{n}(b, c) \geq D_{n}(a, c)$ 을 모두 만족한다. 그리고 (4) 항상 $0 \leq D_{n}(a, b) \leq 1$ 도 성립된다.

[정의1]은 다변량적 일반 표현으로 정의된 것인데, 예를 들어, 이변량 확률밀도함수 $f_{a}\left(x_{1}, x_{2}\right)$ 와 $f_{b}\left(x_{1}, x_{2}\right)$ 의 분포간 거리는 다음과 같이 표현될 수 있다

$$
D_{2}(a, b)=\frac{1}{2} \int_{-\infty}^{\infty} \int_{-\infty}^{\infty}\left|f_{a}\left(x_{1}, x_{2}\right)-f_{b}\left(x_{1}, x_{2}\right)\right| d x_{1} d x_{2}
$$

[정의2] $n$ 변량 정규분포 $N_{n}(\mu, \Sigma)$ 를 따르는 확률변수벡터 $X$ 가 $n$ 차원 타원체(ellipsoid)의 외부 영역인 $H_{n}(\xi, M, \beta)=\left\{\underline{x}:(x-\xi)^{\prime} M(x-\xi) \geq \beta, M\right.$ 은 크기 $n$ 인 양정치행렬, $\beta$ 는 음 아닌 실수 $\}$ 에 속할 
확률을 $P\left\{X \in H_{n}(\xi, M, \beta) ; N_{n}(\underline{\mu}, \Sigma)\right\}$ 라고 표현한다. 그리고 $\underline{\mu}=\varrho$ 이고 $\Sigma=I$ 일 때의 이 확률을 $\Phi_{n}(\xi, M, \beta)$ 라고 표현한다.

$M$ 의 최대고유값을 $\lambda$ 라 하자. 그러면 $H_{n}(\xi, M, \beta)=H_{n}(\xi, M / \lambda, \lambda \beta)$ 이고 $M / \lambda$ 의 최대고유값은 1 이 되기 때문에 우리는 이를 고려하여 $H_{n}(\xi, M, \beta)$ 표현에서 $n$ 을 타원체의 '차원', $\xi$ 를 타원체의 '중 심벡터', $M / \lambda$ 을 타원체의 '모양행렬', $\lambda \beta$ 를 타원체의 '크기값'이라 명명한다.

[성질1] $P\left\{X \in H_{n}(\xi, M, \beta) ; N_{n}(\mu, \Sigma)\right\}=\Phi_{n}\left(\Sigma^{-1 / 2}(\xi-\mu), \Sigma^{1 / 2} M \Sigma^{1 / 2}, \beta\right)$ 이 항상 성립된다.

(증명) $N_{n}(\mu, \Sigma)$ 을 따르는 확률변수벡터 $X$ 의 확률밀도함수를 $\phi(x ; \mu, \Sigma)$ 라고 하자. 그러면 증명과 정은 다음과 같다.

$$
\begin{array}{rlrl} 
& P\left\{X \in H_{n}(\xi, M, \beta) ; N_{n}(\underline{\mu}, \Sigma)\right\} & & \\
= & \int_{H_{n}(\xi, M, \beta)} \phi(x ; \underline{\mu}, \Sigma)(d x), & & \text { [정의2]에 의해 } \\
= & \int_{H_{n}\left(\Sigma^{-1 / 2}(\xi-\mu) \cdot \Sigma^{1 / 2} M \Sigma^{1 / 2}, \beta\right)} \phi(z ; 0, D(d \underline{z}), & & \text { 치환 } \underline{z}=\Sigma^{-1 / 2}(x-\underline{\mu}) \text { 에 의해 } \\
= & P\left\{Z \in H_{n}\left(\Sigma^{-1 / 2}(\xi-\underline{\mu}), \Sigma^{1 / 2} M \Sigma^{1 / 2}, \beta\right) ; N_{n}(0, I)\right\}, & {[\text { 정의2]에 의해 }} \\
= & \Phi_{n}\left(\Sigma^{-1 / 2}(\xi-\underline{\mu}), \Sigma^{1 / 2} M \Sigma^{1 / 2}, \beta\right) & & \text { [정의2]에 의해 }
\end{array}
$$

[성질1]은 어떤 다변량정규분포 $N_{n}(\underline{\mu}, \Sigma)$ 에서라도 주어진 타원체의 외부영역 $H_{n}(\xi, M, \beta)$ 의 확 률은 표준화 변환된 타원체의 외부영역 $H_{n}\left(\Sigma^{-1 / 2}(\xi-\mu), \Sigma^{1 / 2} M \Sigma^{1 / 2}, \beta\right)$ 에 대한 다변량표준정규분포 $N_{n}(0, n)$ 에서의 확률과 같음을 의미하는 것이다.

[성질2] $\alpha<1 / 2$ 일 때 $P\left\{X \in H_{1}\left(\mu, \sigma^{-2}, z_{\alpha}^{2}\right) ; N_{1}\left(\mu, \sigma^{2}\right)\right\}=\Phi_{1}\left(0,1, z_{\alpha}^{2}\right)=2 \alpha$ 가 된다. 여기서, $z_{\alpha}$ 는 $\int_{-\infty}^{z_{a}} \phi(x ; 0,1) d x=1-\alpha$ 를 만족하는 값이다.

(증명) [성질1]에 의해 $P\left\{X \in H_{1}\left(\mu, \sigma^{-2}, z_{\alpha}^{2}\right) ; N_{1}\left(\mu, \sigma^{2}\right)\right\}=\Phi_{1}\left(0,1, z_{\alpha}^{2}\right)$ 가 되고, [정의2]의 $\Phi_{n}(,$,$) 의$ 정의에 의해 $\Phi_{1}\left(0,1, z_{\alpha}^{2}\right)=\int_{x^{\prime} x \geq z_{\alpha}^{2}} \phi(x ; 0,1) d x=2 \alpha$ 가 된다.

$\Phi()$ 가 일변량 표준정규분포의 분포함수(cdf)라면 [성질2]를 통해 $\Phi_{1}(0,1, \beta)=2(1-\Phi(\sqrt{\beta}))$ 가 성립함을 알 수 있다.

[성질3] $\lambda>0$ 이면 $\Phi_{n}(\xi, \lambda M, \beta)=\Phi_{n}(\xi, M, \beta / \lambda)$ 이 항상 성립된다.

(증명) 좌변 $=\int_{(x-\xi)^{\prime}(\lambda M)(x-\xi) \geq \beta} \phi(z ; \ell, I)(d z)=\int_{(x-\xi) M(x-\xi) \geq \beta / \lambda} \phi(z ; 0, D)(d z)=$ 우변

[성질4] $P_{n}$ 이 크기 $n$ 인 직교행렬이라면 $\Phi_{n}(\xi, I, \beta)=\Phi_{n}\left(P_{n} \xi, I, \beta\right)$ 이 항상 성립한다.

(증명) $P_{n}$ 가 직교행렬이면 $y=P_{n}$ 라는 치환에서 $\left(d_{x}\right)=(d x)$ 가 된다는 성질을 이용하면 다음 에 의해 쉽게 증명된다. 


$$
\begin{aligned}
\Phi_{n}(\xi, I, \beta) & =\int_{\left(x-\xi^{\prime}(x-\xi) \geq \beta\right.} \phi(x ; 0, I)(d x) \\
& =\int_{\left(P_{n}^{\prime} y-\xi\right)^{\prime}\left(P_{n}^{\prime} y-\xi\right) \geq \beta} \phi\left(y ; 0, D(d y), \text { 치환 } y=P_{n} x\right. \text { 에 의해 } \\
& =\int_{\left(y-P_{n} \xi \xi^{\prime}\left(y-P_{n} \xi\right) \geq \beta\right.} \phi(y ; 0, D)(d y) \\
& =\Phi_{n}\left(P_{n} \xi, I, \beta\right)
\end{aligned}
$$

이 [성질4]는 다른 표현으로는 $\left\ulcorner\xi \xi=\eta^{\prime} \eta\right.$ 이면 $\Phi_{n}(\xi, I, \beta)=\Phi_{n}(\eta, I, \beta)$ 이 항상 성립」 가 되는데 이는 $n$ 변량 표준정규분포에서 반경이 정해진 $n$ 차원 초구(hyper-sphere)영역 외부의 확률은 그 초구의 중심 좌표값들 개개에 의존한다기 보다는 원점에서 중심까지의 거리에만 의존함을 의미한 다. 이에 본 연구에서는 편의상 $n=P_{n} \xi=(\sqrt{\xi \xi} 0 \cdots 0)^{\prime}$ '을 이용할 것이며, $\sqrt{\xi \xi}$ 을 초구의 '중심 거리값'이라 한다.

\section{3.두 다변량정규분포 확률밀도함수 그래프들의 관계와 이들의 분포간 거리}

본 절에서는 차원이 동일한 두 다변량정규분포 확률밀도함수 그래프들의 교점에 관해 [정리1]에 서 먼저 살펴보고, 이를 이용하여 [정의1]에서의 '분포간 거리' 정의에 따라 두 다변량정규분포들 사이의 분포간 거리를 [정리2]와 [정리3]에서 밝힌다. [정리2]는 공분산행렬이 동일한 경우의 결과 이며, [정리3]은 공분산행렬이 서로 다른 경우의 결과이다. 그리고 [따름정리1], [따름정리2]를 통 해 일변량 정규분포에 적용된 [정리1] [정리3]의 유도과정과 결과를 제공한다. 이에는 교육적 목 적을 위해 행렬표현을 사용하지 않았다.

[보조정리]

$$
\begin{aligned}
& \left(\underline{\mu}_{b}^{\prime} \Sigma_{b}^{-1}-\underline{\mu}_{a}^{\prime} \Sigma_{a}^{-1}\right)\left(\Sigma_{b}^{-1}-\Sigma_{a}^{-1}\right)^{-1}\left(\Sigma_{b}^{-1} \underline{\mu}_{b}-\Sigma_{a}^{-1} \underline{\mu}_{a}\right)-\left(\underline{\mu}_{b}^{\prime} \Sigma_{b}^{-1} \underline{\mu}_{b}-\underline{\mu}_{a}^{\prime} \Sigma_{a}^{-1} \underline{\mu}_{a}\right) \\
& =\left(\underline{\mu}_{b}-\underline{\mu}_{a}\right)^{\prime}\left(\Sigma_{a}-\Sigma_{b}\right)^{-1}\left(\underline{\mu}_{b}-\underline{\mu}_{a}\right)
\end{aligned}
$$

(증명) 행렬 $A, B$ 는 각각 크기가 $p \times p, q \times q$ 인 정칙(nonsingular)행렬이고, 행렬 $C, D$ 는 각각 크 기가 $p \times q, q \times p$ 라고 하자. 그러면 $Q=A+C B D$ 일 때 다음 식(1)이 항상 성립된다(Muirhead, 1982. 580쪽 참조).

$$
Q^{-1}=A^{-1}-A^{-1} C B\left(B+B D A^{-1} C B\right)^{-1} B D A^{-1}
$$

이 식(1)를 이용하면 다음 식(2)의 관계식들을 얻을 수 있다.

$$
\left\{\Sigma_{b}-\Sigma_{b} \Sigma_{a}^{-1} \Sigma_{b}\right\}^{-1}=\Sigma_{b}^{-1}-\left(\Sigma_{b}-\Sigma_{a}\right)^{-1}, \quad\left\{\Sigma_{a}-\Sigma_{a} \Sigma_{b}^{-1} \Sigma_{a}\right\}^{-1}=\Sigma_{a}^{-1}+\left(\Sigma_{b}-\Sigma_{a}\right)^{-1}
$$

따라서

$$
\begin{aligned}
& \text { 좌변 }=\left(\underline{\mu}_{b}^{\prime} \Sigma_{b}^{-1}-\underline{\mu}_{a}^{\prime} \Sigma_{a}^{-1}\right)\left(\Sigma_{b}^{-1}-\Sigma_{a}^{-1}\right)^{-1}\left(\Sigma_{b}^{-1} \underline{\mu}_{b}-\Sigma_{a}^{-1} \underline{\mu}_{a}\right)-\left(\underline{\mu}_{b}^{\prime} \Sigma_{b}^{-1} \underline{\mu}_{b}-\underline{\mu}_{a}^{\prime} \Sigma_{a}^{-1} \underline{\mu}_{a}\right) \\
& =\mu_{b}^{\prime} \Sigma_{b}^{-1}\left(\Sigma_{b}^{-1}-\Sigma_{a}^{-1}\right)^{-1} \Sigma_{b}^{-1} \underline{\mu}_{b}-\mu_{b}^{\prime} \Sigma_{b}^{-1}\left(\Sigma_{b}^{-1}-\Sigma_{a}^{-1}\right)^{-1} \Sigma_{a}^{-1} \mu_{a}-\mu_{a}^{\prime} \Sigma_{a}^{-1}\left(\Sigma_{b}^{-1}-\Sigma_{a}^{-1}\right)^{-1} \Sigma_{b}^{-1} \mu_{b} \\
& +\underline{\mu}_{a}^{\prime} \Sigma_{a}^{-1}\left(\Sigma_{b}^{-1}-\Sigma_{a}^{-1}\right)^{-1} \Sigma_{a}^{-1} \underline{\mu}_{a}-\left(\underline{\mu}_{b}^{\prime} \Sigma_{b}^{-1} \underline{\mu}_{b}-\underline{\mu}_{a}^{\prime} \Sigma_{a}^{-1} \underline{\mu}_{a}\right) \\
& =\underline{\mu}_{b}\left\{\Sigma_{b}-\Sigma_{b} \Sigma_{a}^{-1} \Sigma_{b}\right\}^{-1} \underline{\mu}_{b}-\underline{\mu}_{b}\left\{\Sigma_{a}-\Sigma_{b}\right\}^{-1} \underline{\mu}_{a} \\
& -\underline{\mu}_{a}^{\prime}\left\{\Sigma_{a}-\Sigma_{b}\right\}^{-1} \underline{\mu}_{b}+\underline{\mu}_{a}^{\prime}\left\{\Sigma_{a} \Sigma_{b}{ }^{1} \Sigma_{a}-\Sigma_{a}\right\}^{-1} \underline{\mu}_{a}-\left(\underline{\mu}_{b}^{\prime} \Sigma_{b}^{-1} \underline{\mu}_{b}-\underline{\mu}_{a}^{\prime} \Sigma_{a}^{-1} \underline{\mu}_{a}\right) \\
& =\underline{\mu}_{b}^{\prime}\left\{\Sigma_{b}^{-1}-\left(\Sigma_{b}-\Sigma_{a}\right)^{-1} \underline{\mu}_{b}-\underline{\mu}_{b}^{\prime}\left\{\Sigma_{a}-\Sigma_{b}\right\}^{-1} \underline{\mu}_{a}-\underline{\mu}_{a}^{\prime}\left\{\Sigma_{a}-\Sigma_{b}\right\}^{-1} \underline{\mu}_{b} \quad\right. \text { 식(2)에 의해 } \\
& -\mu_{a} \cdot\left\{\Sigma_{a}^{-1}+\left(\Sigma_{b}-\Sigma_{a}\right)^{-1} \underline{\mu}_{a}-\left(\mu_{b}^{\prime} \Sigma_{b}^{-1} \underline{\mu}_{b}-\mu_{a}^{\prime} \Sigma_{a}^{-1} \mu_{a}\right)\right.
\end{aligned}
$$


A Study on Volume of Difference of Two Joint pdf's, 753

$$
\begin{aligned}
& =\underline{\mu}_{b}^{\prime} \Sigma_{b}^{-1} \underline{\mu}_{b}-\mu_{b}^{\prime}\left(\Sigma_{b}-\Sigma_{a}\right)^{-1} \underline{\mu}_{b}+\underline{\mu}_{b}^{\prime}\left\{\Sigma_{b}-\Sigma_{a}\right\}^{-1} \mu_{a}+\underline{\mu}_{a}\left\{\Sigma_{b}-\Sigma_{a}\right\}^{-1} \underline{\mu}_{b} \\
& -\mu_{a}^{\prime} \Sigma_{a}^{-1} \mu_{a}-\underline{\mu}_{a}^{\prime}\left(\Sigma_{b}-\Sigma_{a}\right)^{-1} \mu_{a}-\left(\mu_{b}^{\prime} \Sigma_{b}^{-1} \mu_{b}-\mu_{a}^{\prime} \Sigma_{a}^{-1} \mu_{a}\right) \\
& =-\underline{\mu}_{b}^{\prime}\left(\Sigma_{b}-\Sigma_{a}\right)^{-1} \underline{\mu}_{b}+\underline{\mu}_{b}\left\{\Sigma_{b}-\Sigma_{a}\right\}^{-1} \underline{\mu}_{a}+\underline{\mu}_{a}\left\{\Sigma_{b}-\Sigma_{a}\right\}^{-1} \underline{\mu}_{b}-\underline{\mu}_{a}^{\prime}\left(\Sigma_{b}-\Sigma_{a}\right)^{-1} \underline{\mu}_{a} \\
& =\left(\underline{\mu}_{b}-\underline{\mu}_{a}\right)^{\prime}\left(\Sigma_{a}-\Sigma_{b}\right)^{-1}\left(\underline{\mu}_{b}-\underline{\mu}_{a}\right) \\
& =\text { 우변 }
\end{aligned}
$$

[정리1] $X_{a} \sim N_{n}\left(\mu_{a}, \Sigma_{a}\right), X_{b} \sim N_{n}\left(\mu_{b}, \Sigma_{b}\right)$ 의 확률밀도함수들 $\phi_{a}\left(x ; \mu_{a}, \Sigma_{a}\right), \phi_{b}\left(x ; \mu_{b}, \Sigma_{b}\right)$ 에 대하 여 부등식 $\phi_{a}\left(x ; \underline{\mu}_{a}, \Sigma_{a}\right) \geq \phi_{b}\left(x ; \mu_{b}, \Sigma_{b}\right)$ 의 해집합을 $C_{n}\left(\phi_{a} \geq \phi_{b}\right)$ 라고 표현한다면 다음의 (i)와 (ii) 가 성립된다.

(i) $\Sigma_{a}=\Sigma_{b}(=\Sigma)$ 일 때, $C_{n}\left(\phi_{a} \geq \phi_{b}\right)=\left\{x \mid 2\left(\underline{\mu}_{b}-\underline{\mu}_{a}\right)^{\prime} \Sigma^{-1} x \leq \underline{\mu}_{b}^{\prime} \Sigma^{-1} \underline{\mu}_{b}-\underline{\mu}_{a}^{\prime} \Sigma^{-1} \underline{\mu}_{a}\right\}$

(ii) $\Sigma_{a} \neq \Sigma_{b}$ 일 때, $C_{n}\left(\phi_{a} \geq \phi_{b}\right)=\left\{x \mid(x-\alpha)^{\prime} A(x-\alpha) \geq \beta\right\}$

여기서, $A=\Sigma_{b}^{-1}-\Sigma_{a}^{-1}, \underline{\alpha}=A^{-1}\left(\Sigma_{b}^{-1} \underline{\mu}_{b}-\Sigma_{a}^{-1} \underline{\mu}_{a}\right)$, $\beta=\left(\underline{\mu}_{b}-\underline{\mu}_{a}\right)^{\prime}\left(\Sigma_{a}-\Sigma_{b}\right)^{-1}\left(\underline{\mu}_{b}-\underline{\mu}_{a}\right)+\left(\log \left|\Sigma_{a}\right|-\log \left|\Sigma_{b}\right|\right)$ 이다.

(증명)

$$
\begin{aligned}
& \phi_{a}\left(\underline{x} ; \underline{\mu}_{a}, \Sigma_{a}\right) \geq \phi_{b}\left(\underline{\underline{x}} ; \underline{\mu}_{b}, \Sigma_{b}\right) \\
\Leftrightarrow & (2 \pi)^{-n / 2}\left|\Sigma_{a}\right|^{-1 / 2} \exp \left[-\left(\underline{x}-\underline{\mu}_{a}\right)^{\prime} \Sigma_{a}^{-1}\left(\underline{x}-\underline{\mu}_{a}\right) / 2\right]
\end{aligned}
$$$$
\geq(2 \pi)^{-n / 2}\left|\Sigma_{b}\right|^{-1 / 2} \exp \left[-\left(\underline{x}-\underline{\mu}_{b}\right)^{\prime} \Sigma_{b}^{-1}\left(\underline{x}-\underline{\mu}_{b}\right) / 2\right]
$$$$
\Leftrightarrow\left(x-\mu_{b}\right)^{\prime} \Sigma_{b}^{-1}\left(x-\mu_{b}\right)-\left(\underline{x}-\underline{\mu}_{a}\right)^{\prime} \Sigma_{a}^{-1}\left(x-\mu_{a}\right) \geq \log \left(\left|\Sigma_{a}\right| /\left|\Sigma_{b}\right|\right)
$$$$
\Leftrightarrow x^{\prime} \Sigma_{b}^{-1} x-\underline{\mu}_{b}^{\prime} \Sigma_{b}^{-1} x-x^{\prime} \Sigma_{b}^{-1} \underline{\mu}_{b}+\underline{\mu}_{b}^{\prime} \Sigma_{b}^{-1} \underline{\mu}_{b}
$$$$
-\underline{x}^{\prime} \Sigma_{a}^{-1} x+\mu_{a}^{\prime} \Sigma_{a}^{-1} x+\underline{x}^{\prime} \Sigma_{a}^{-1} \underline{\mu}_{a}-\underline{\mu}_{a}^{\prime} \Sigma_{a}^{-1} \underline{\mu}_{a} \geq \log \left(\left|\Sigma_{a}\right| /\left|\Sigma_{b}\right|\right)
$$$$
\Leftrightarrow x^{\prime}\left(\Sigma_{b}^{-1}-\Sigma_{a}^{-1}\right) \underline{x}-\left(\mu_{b}^{\prime} \Sigma_{b}^{-1}-\mu_{a}^{\prime} \Sigma_{a}^{-1}\right) \underline{x}
$$$$
-\underline{x}^{\prime}\left(\Sigma_{b}^{-1} \underline{\mu}_{b}-\Sigma_{a}^{-1} \underline{\mu}_{a}\right)+\left(\underline{\mu}_{b}^{\prime} \Sigma_{b}^{-1} \underline{\mu}_{b}-\underline{\mu}_{a}^{\prime} \Sigma_{a}^{-1} \underline{\mu}_{a}\right) \geq \log \left(\left|\Sigma_{a}\right| /\left|\Sigma_{b}\right|\right)
$$

윗 식(3)에서 $\Sigma_{a}=\Sigma_{b}=\Sigma$ 을 대입하면 (i)이 증명된다. 한편 $\Sigma_{a} \neq \Sigma_{b}$ 일 때의 윗 부등식(3)는 다시 아래와 같이 재표현될 수 있는데 이의 우변에 [보조정리]의 결과를 적용하면 (ii)가 증명된다.

$$
\begin{aligned}
& \left\{x-\left(\Sigma_{b}^{-1}-\Sigma_{a}^{-1}\right)^{-1}\left(\Sigma_{b}^{-1} \underline{\mu}_{b}-\Sigma_{a}^{-1} \underline{\mu}_{a}\right)\right\}^{\prime}\left(\Sigma_{b}^{-1}-\Sigma_{a}^{-1}\right)\left\{x-\left(\Sigma_{b}^{-1}-\Sigma_{a}^{-1}\right)^{-1}\left(\Sigma_{b}^{-1} \underline{\mu}_{b}-\Sigma_{a}^{-1} \underline{\mu}_{a}\right)\right\} \\
& z\left(\underline{\mu}_{b}^{\prime} \Sigma_{b}^{-1}-\underline{\mu}_{a}^{\prime} \Sigma_{a}^{-1}\right)\left(\Sigma_{b}^{-1}-\Sigma_{a}^{-1}\right)^{-1}\left(\Sigma_{b}^{-1} \underline{\mu}_{b}-\Sigma_{a}^{-1} \underline{\mu}_{a}\right)-\left(\underline{\mu}_{b}^{\prime} \Sigma_{b}^{-1} \underline{\mu}_{b}-\underline{\mu}_{a}^{\prime} \Sigma_{a}^{-1} \underline{\mu}_{a}\right)+\log \left(\left|\Sigma_{a}\right|\right)-\log \left(\left|\Sigma_{b}\right|\right)
\end{aligned}
$$

참고로 [정리1]-(i)의 결과는 공분산구조가 같은 두 개의 $n$ 변량정규분포 확률밀도함수 그래프들 의 교집합은 평균벡터까지 일치하지 않은 한 $(n-1)$ 차원 초평면(hyper-plane)이 됨을 의미한다. 그리고 [정리1]-(ii)의 결과는 공분산구조가 다르되 $\left(\Sigma_{a}-\Sigma_{b}\right)$ 가 양정치(positive definite) 또는 음 정치(negative definite) 행렬이 되면 $n$ 변량정규분포 확률밀도함수 그래프들의 교집합은 $n$ 차원 타 원체가 됨을 의미한다.

[정리2] $X_{a} \sim N_{n}\left(\mu_{a}, \Sigma_{a}\right), X_{b} \sim N_{n}\left(\mu_{b}, \Sigma_{b}\right)$ 이고, $\Sigma_{a}=\Sigma_{b}(=\Sigma)$ 일 때 다음의 사실들이 성립된다.

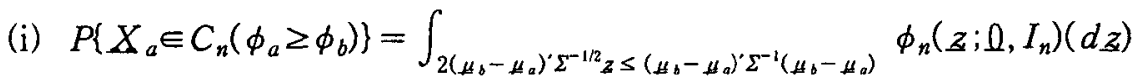

$$
P\left\{X_{b} \in C_{n}\left(\phi_{a} \geq \phi_{b}\right)\right\}=\int_{2\left(\mu_{b}-\underline{\mu}_{a}\right) \cdot \Sigma^{-1 / 2} z \leq-\left(\underline{\mu}_{b}-\underline{\mu}_{a}\right) \Sigma^{-1}\left(\underline{\mu}_{b}-\underline{\mu}_{a}\right)} \phi_{n}\left(\underline{z} ; \underline{0}, I_{n}\right)(d \underline{z})
$$

(ii) 특히 $\underline{\mu}_{a}=u 1_{n}, \underline{\mu}_{b}=v 1_{n}, \quad \Sigma=k^{2} I_{n}(u, v, k$ 는 $u\langle v, k>0$ 인 상수)이라면 다음이 성립된 
다. 여기서 $\Phi()$ 은 일변량 표준정규분포의 분포함수 $(\mathrm{cdf})$ 이다.

$$
P\left\{\underline{X}_{a} \in C_{n}\left(\phi_{a} \geq \phi_{b}\right)\right\}=\Phi(\sqrt{n}(v-u) / 2 k), \quad P\left\{\underline{X}_{b} \in C_{n}\left(\phi_{a} \geq \phi_{b}\right)\right\}=\Phi(-\sqrt{n}(v-u) / 2 k)
$$

(iii) 따라서 (ii)의 조건하에서는 다음이 성립된다. $\quad D_{n}(a, b)=2 \Phi(\sqrt{n}(v-u) / 2 k)-1$

(증명) [정리1]의 (i)에 의하면 $C_{n}\left(\phi_{a} \geq \phi_{b}\right)=\left\{x \mid 2\left(\mu_{b}-\mu_{a}\right)^{\prime} \Sigma^{-1} x \leq \mu_{b}^{\prime} \Sigma^{-1} \mu_{b}-\mu_{a}^{\prime} \Sigma^{-1} \underline{\mu}_{a}\right\}$ 가 된다. 따라서 다음의 식(4), 식(5)에 의해 (i)이 증명된다.

$$
\begin{aligned}
& P\left\{\underline{X}_{a} \in C_{n}\left(\phi_{a} \geq \phi_{b}\right)\right\} \\
& =P\left\{\underline{X}_{a}^{a} \in C_{n}\left(\phi_{a} \geq \phi_{b}\right) ; N_{n}\left(\mu_{a} \Sigma\right)\right\} \\
& =\int_{2\left(\mu_{b}-\mu_{a}\right) \Sigma^{-1} x \leq \psi_{b} \Sigma^{-1} \mu_{b}-\mu_{a} \Sigma^{-1} \mu_{a}} \phi_{n}\left(x ; \underline{\mu}_{a}, \Sigma\right)(d x), \quad \text { [정리1] } \text { 의 }(i i) \text { 에 의해 } \\
& =\int_{2\left(\mu_{b}-\mu_{a}\right) \Sigma^{-1 / 2} z_{z} \leq\left(\mu_{b}-\mu_{a}\right) \Sigma^{-1}\left(\mu_{b}-\mu_{o}\right)} \phi_{n}\left(\underline{z} ; 0, I_{n}\right)(d \underline{z}) \text {, 치환 } x=\Sigma^{1 / 2} \underline{z}+\underline{\mu}_{a} \text { 에 의해 } \\
& P\left\{\underline{X}_{b} \in C_{n}\left(\phi_{a} \geq \phi_{b}\right)\right\} \\
& =P\left\{\underline{X}_{b} \in C_{n}\left(\phi_{a} \geq \phi_{b}\right) ; N_{n}\left(\underline{\mu}_{b}, \Sigma\right)\right\} \\
& =\int_{2\left(\mu_{b}-\mu_{a}\right) \Sigma^{-1} x \leq \mu_{b}^{\prime} \Sigma^{-1} \mu_{b}-\mu_{a} \Sigma^{-1} \mu_{a}} \phi_{n}\left(x ; \underline{\mu}_{b}, \Sigma\right)(d x), \quad \text { [정리1]의 (ii)에 의해 } \\
& =\int_{2\left(\mu_{b}-\mu_{a}\right) \Sigma^{1 / 2} z \leq-\left(\mu_{b}-\mu_{a}\right) \Sigma^{-1}\left(\mu_{b}-\mu_{a}\right)} \phi_{n}\left(\underline{z} ; 0, I_{n}\right)(d z) \text {, 치환 } x=\Sigma^{1 / 2} \underline{z}+\underline{\mu}_{b} \text { 에 의해 }
\end{aligned}
$$

식(4)와 식(5)에서 $\underline{\mu}_{a}=u \perp_{n}, \underline{\mu}_{b}=v 1_{n}, \quad \Sigma=k^{2} I_{n}(u, v, k$ 는 $v>u, k>0$ 인 상수)이라면, 그리고 $Z_{1}, Z_{2}, \cdots, Z_{n}$ 이 표준정규분포로부터 얻어진 확률표본인 경우 $\left(Z_{1}+Z_{2}+\cdots+Z_{n}\right) / \sqrt{n}$ 은 표준정규분포 를 따른다는 사실을 이용하면 (ii)를 증명하는 다음의 전개과정들을 얻을 수 있다.

$$
\begin{aligned}
& P\left\{X_{a} \in C_{n}\left(\phi_{a} \geq \phi_{b}\right)\right\}=\int_{I^{\prime} z / \sqrt{n} \leq \sqrt{n}(v-u) / 2 k} \phi_{n}\left(z ; 0, I_{n}\right)(d z)=\emptyset(\sqrt{n}(v-u) / 2 k), \\
& P\left\{X_{b} \in C_{n}\left(\phi_{a} \geq \phi_{b}\right)\right\}=\int_{I^{\prime} z / \sqrt{n} \leq-\sqrt{n}(v-u) / 2 k} \phi_{n}\left(z ; 0, I_{n}\right)(d z)=\emptyset(-\sqrt{n}(v-u) / 2 k)
\end{aligned}
$$

그리고 (iii)은 다음에 의해 쉽게 증명된다.

$$
\begin{aligned}
D_{n}(a, b)= & 1 / 2\left\{\left[P\left\{X_{a} \in C_{n}\left(\phi_{a} \geq \phi_{b}\right)\right\}-P\left\{X_{b} \in C_{n}\left(\phi_{a} \geq \phi_{b}\right)\right\}\right], \quad[\text { 정의1]에 의해 }\right. \\
& \left.+\left[P\left\{X_{b} \in C_{n}\left(\phi_{a} \leq \phi_{b}\right)\right\}-P\left\{X_{a} \in C_{n}\left(\phi_{a} \leq \phi_{b}\right)\right\}\right]\right\} \\
= & 1 / 2\left\{\left[P\left\{X_{a} \in C_{n}\left(\phi_{a} \geq \phi_{b}\right)\right\}-P\left\{X_{b} \in C_{n}\left(\phi_{a} \geq \phi_{b}\right)\right\}\right]\right. \\
& \left.\quad+\left[\left\{1-P\left\{X_{b} \in C_{n}\left(\phi_{a} \geq \phi_{b}\right)\right\}\right\}-\left\{1-P\left\{X_{a} \in C_{n}\left(\phi_{a} \geq \phi_{b}\right)\right\}\right\}\right]\right\} \\
= & P\left\{X a \in C_{n}\left(\phi_{a} \geq \phi_{b}\right)\right\}-P\left\{X_{b} \in C_{n}\left(\phi_{a} \geq \phi_{b}\right)\right\} \\
= & \Phi(\sqrt{n}(v-u) / 2 k)-\Phi(-\sqrt{n}(v-u) / 2 k) \\
= & 2 \Phi(\sqrt{n}(v-u) / 2 k)-1, \quad \text { 성질 } \Phi(-z)=1-\Phi(z) \text { 을 이용하면 }
\end{aligned}
$$

[정리3] $X_{a} \sim N_{n}\left(\underline{\mu}_{a}, \Sigma_{a}\right), X_{b} \sim N_{n}\left(\underline{\mu}_{b}, \Sigma_{b}\right)$ 이고, $\left(\Sigma_{a}-\Sigma_{b}\right)$ 가 양정치행렬일 때 다음의 사실들이 성립된다.

(i) $C_{n}\left(\phi_{a} \geq \phi_{b}\right)=\left\{x \mid \phi_{a}\left(x ; \underline{\mu}_{a}, \Sigma_{a}\right) \geq \phi_{b}\left(x ; \underline{u}_{b}, \Sigma_{b}\right)\right\}=H_{n}(\underline{\alpha}, A, \beta)$

여기서, $\alpha, A, \beta$ 는 [정리1]의 (ii)에 주어진 것과 같다.

(ii) $P\left\{X_{a} \in C_{n}\left(\phi_{a} \geq \phi_{b}\right)\right\}=\Phi_{n}\left(\eta_{a}, \Sigma_{a}^{1 / 2} \Sigma_{b}^{-1} \Sigma_{a}^{1 / 2}-I, \beta\right)$ 


$$
\begin{aligned}
& P\left\{X_{b} \in C_{n}\left(\phi_{a} \geq \phi_{b}\right)\right\}=\Phi_{n}\left(\eta_{b}, I-\Sigma_{b}^{1 / 2} \Sigma_{a}^{-1} \Sigma_{b}^{1 / 2}, \beta\right)
\end{aligned}
$$

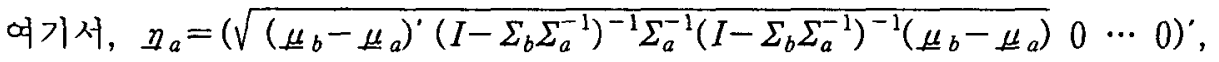

$$
\begin{aligned}
& \eta_{b}=\left(\sqrt{\left(\mu_{a}-\mu_{b}\right)^{\prime}\left(I-\Sigma_{a} \Sigma_{b}^{-1}\right)^{-1} \Sigma_{b}^{-1}\left(I-\Sigma_{a} \Sigma_{b}^{-1}\right)^{-1}\left(\mu_{a}-\mu_{b}\right)} 0 \cdots 0\right)^{\prime} \text { 이다. }
\end{aligned}
$$

(iii) 특히 $\mu_{a}=\mu_{b}$ 라면 다음이 성립되는데 이는 모두 $\Sigma_{a}^{-1 / 2} \Sigma_{b}^{1 / 2}$ 의 함수가 된다.

$$
\begin{aligned}
& P\left\{X_{a} \in C_{n}\left(\phi_{a} \geq \phi_{b}\right)\right\}=\Phi_{n}\left(0, \Sigma_{a}^{1 / 2} \Sigma_{b}^{-1} \Sigma_{a}^{1 / 2}-I, \log \left|\Sigma_{a} \Sigma_{b}^{-1}\right|\right) \\
& P\left(X_{b} \in C_{n}\left(\phi_{a} \geq \phi_{b}\right)\right\}=\Phi_{n}\left(0, I-\Sigma_{b}^{1 / 2} \Sigma_{a}^{-1} \Sigma_{b}^{1 / 2}, \log \left|\Sigma_{a} \Sigma_{b}^{-1}\right|\right) \\
& \text { (iv) } D_{n}(a, b)=\Phi_{n}\left(\eta_{a}, \Sigma_{a}^{1 / 2} \Sigma_{b}^{-1} \Sigma_{a}^{1 / 2}-I, \beta\right)-\Phi_{n}\left(\eta_{b}, I-\Sigma_{b}^{1 / 2} \Sigma_{a}^{-1} \Sigma_{b}^{1 / 2}, \beta\right)
\end{aligned}
$$

(증명) $\left(\Sigma_{a}-\Sigma_{b}\right)$ 가 양정치행렬이라면 $\left(\Sigma_{b}^{-1}-\Sigma_{a}^{-1}\right)$ 도 양정치행렬이 된다는 사실을 이용하면 $C_{n}\left(\phi_{a} \geq \phi_{b}\right)$ 는 [정리1]의 (ii)에 의하면 $n$ 차원 타원체의 외부영역이 되고, 이는 [정의2]에 주어진 $H_{n}(,$, ) 의 조건을 만족하기 때문에 [정의2]에 의해 (i)이 중명된다. 한편 (ii)의 첫 번째 관계식은 아래 식(6)의 두 관계식을 이용하면 다음과 같이 증명된다.

$$
\begin{aligned}
& \underline{\alpha}-\underline{\mu}_{a}=\left(I-\Sigma_{b} \Sigma_{a}^{-1}\right)^{-1}\left(\underline{\mu}_{b}-\underline{\mu}_{a}\right), \quad \Sigma_{a}^{1 / 2} A \Sigma_{a}^{1 / 2}=\Sigma_{a}^{1 / 2} \Sigma_{b}^{-1} \Sigma_{a}^{1 / 2}-I \\
& P\left\{X_{a} \in C_{n}\left(\phi_{a} \geq \phi_{b}\right)\right\}=P\left\{X_{a} \in H_{n}(\alpha, A, \beta) ; N_{n}\left(\mu_{a}, \Sigma_{a}\right)\right\} \\
& =P\left\{Z \in H_{n}\left(\Sigma_{a}^{-1 / 2}\left(\alpha-\mu_{a}\right), \Sigma_{a}^{1 / 2} A \Sigma_{a}^{1 / 2}, \beta\right) ; N_{n}(0, D)\right\}, \quad[\text { 성질1]에 의해 } \\
& =\emptyset_{n}\left(\Sigma_{a}^{-1 / 2}\left(\alpha-\mu_{a}\right), \Sigma_{a}^{1 / 2} A \Sigma_{a}^{1 / 2}, \beta\right) \\
& =\Phi_{n}\left(\Sigma_{a}^{-1 / 2}\left(I-\Sigma_{b} \Sigma_{a}^{-1}\right)^{-1}\left(\underline{\mu}_{b}-\underline{\mu}_{a}\right), \Sigma_{a}^{1 / 2} A \Sigma_{a}^{1 / 2}, \beta\right) \text {, 식(6)에 의해 } \\
& =\Phi_{n}\left(\underline{\eta}_{a}, \Sigma_{a}^{1 / 2} A \Sigma_{a}^{1 / 2}, \beta\right) \text {, } \\
& =\Phi_{n}\left(\eta_{a}, \Sigma_{a}^{1 / 2} \Sigma_{b}^{-1} \Sigma_{a}^{1 / 2}-I, \beta\right) \text {, } \\
& \text { [성질4]에 의해 } \\
& \text { 식(6) 의해 }
\end{aligned}
$$

비슷한 과정에 의해 (ii)의 두 번째 관계식도 증명될 수 있다. (iii)은 (ii)의 특수한 경우로 이의 증명과정은 필요치 않겠다. (iv)는 다음에 의해 쉽게 증명된다.

$$
\begin{array}{rlr}
D_{n}(a, b)= & 1 / 2\left\{\left[P\left\{X_{a} \in C_{n}\left(\phi_{a} \geq \phi_{b}\right)\right\}-P\left\{X_{b} \in C_{n}\left(\phi_{a} \geq \phi_{b}\right)\right\}\right]\right. \\
& \left.+\left[P\left\{X_{b} \in C_{n}\left(\phi_{a} \leq \phi_{b}\right)\right\}-P\left(X_{a} \in C_{n}\left(\phi_{a} \leq \phi_{b}\right)\right\}\right]\right\} & \text { [정의1]에 의해 } \\
= & {\left[P\left\{X_{a} \in C_{n}\left(\phi_{a} \geq \phi_{b}\right)\right\}-P\left\{X_{b} \in C_{n}\left(\phi_{a} \geq \phi_{b}\right)\right\}\right]} \\
= & \Phi_{n}\left(\eta_{a}, \Sigma_{a}^{1 / 2} \Sigma_{b}^{-1} \Sigma_{a}^{1 / 2}-I, \beta\right)-\Phi_{n}\left(\underline{\eta}_{b}, I-\Sigma_{b}^{1 / 2} \Sigma_{a}^{-1} \Sigma_{b}^{1 / 2}, \beta\right), \quad[\text { 정리3]의 (ii)에 의해 }
\end{array}
$$

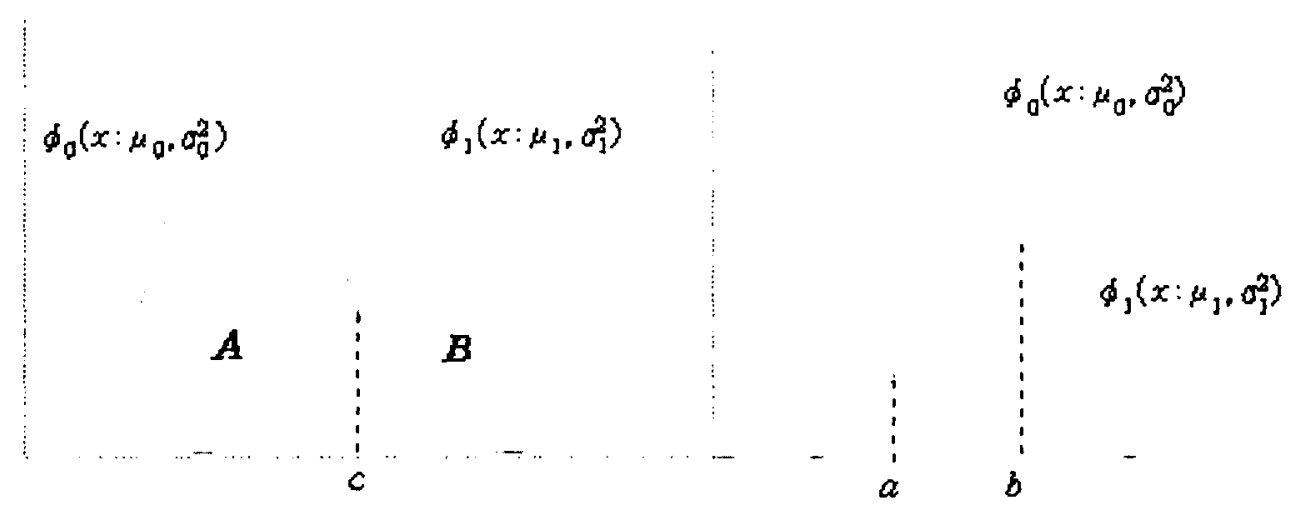

[그립2] 두 일변량정규분포의 확률밀도함수 그래프들의 관계 
[따름정리1] $N\left(\mu_{0}, \sigma_{0}^{2}\right)$ 와 $N\left(\mu_{1}, \sigma_{1}^{2}\right)$ 의 확률밀도함수를 각각 $\phi_{0}\left(x ; \mu_{0}, \sigma_{0}^{2}\right), \phi_{1}\left(x ; \mu_{1}, \sigma_{1}^{2}\right)$ 라 하자. 그러 면 두 확률밀도함수의 곡선은 i) $\mu_{0}=\mu_{1}, \sigma_{0}^{2}=\sigma_{1}^{2}$ 이면 완전히 일치하고, ii) $\mu_{0} \neq \mu_{1}, \sigma_{0}^{2}=\sigma_{1}^{2}$ 이면 오 직 한 점 $c=\left(\mu_{0}+\mu_{1}\right) / 2$ 에서만 만나며, iii) $\sigma_{0}^{2} \neq \sigma_{1}^{2}$ 이면 다음의 서로 다른 두 점 $a, b(a<b)$ 에서 만난다([그림2] 참조).

$$
\begin{aligned}
& a=\frac{\mu_{0} \sigma_{1}^{2}-\mu_{1} \sigma_{0}^{2}-\sigma_{0} \sigma_{1} \sqrt{\left(\mu_{1}-\mu_{0}\right)^{2}+\left(\sigma_{1}^{2}-\sigma_{0}^{2}\right)\left(\log \sigma_{1}^{2}-\log \sigma_{0}^{2}\right)}}{\sigma_{1}^{2}-\sigma_{0}^{2}} \\
& b=\frac{\mu_{0} \sigma_{1}^{2}-\mu_{1} \sigma_{0}^{2}+\sigma_{0} \sigma_{1} \sqrt{\left(\mu_{1}-\mu_{0}\right)^{2}+\left(\sigma_{1}^{2}-\sigma_{0}^{2}\right)\left(\log \sigma_{1}^{2}-\log \sigma_{0}^{2}\right)}}{\sigma_{1}^{2}-\sigma_{0}^{2}}
\end{aligned}
$$

(증명) i)는 자명하므로 ii)와 iii)만 중명한다. [정리1]의 결과를 이용하여 증명할 수 있지만 이해를 위해 행렬표현이 없는 방식으로 직접 증명을 해 본다.

$$
\begin{aligned}
& \phi_{0}(x)=\phi_{1}(x) \\
\Leftrightarrow & \left(\sqrt{2 \pi} \sigma_{0}\right)^{-1} \exp \left\{-\left(x-\mu_{0}\right)^{2} / 2 \sigma_{0}^{2}\right\}=\left(\sqrt{2 \pi} \sigma_{1}\right)^{-1} \exp \left\{-\left(x-\mu_{1}\right)^{2} / 2 \sigma_{1}^{2}\right\} \\
\Leftrightarrow & \sigma_{1} / \sigma_{0}=\exp \left\{\left(x-\mu_{0}\right)^{2} / 2 \sigma_{0}^{2}-\left(x-\mu_{1}\right)^{2} / 2 \sigma_{0}^{2}\right\} \\
\Leftrightarrow & \log \left(\sigma_{1}^{2} / \sigma_{0}^{2}\right)=\left\{\sigma_{1}^{2}\left(x-\mu_{0}\right)^{2}-\sigma_{0}^{2}\left(x-\mu_{1}\right)^{2}\right\} / \sigma_{0}^{2} \sigma_{1}^{2} \\
\Leftrightarrow & \left(\sigma_{1}^{2}-\sigma_{0}^{2}\right) x^{2}-2\left(\mu_{0} \sigma_{1}^{2}-\mu_{1} \sigma_{0}^{2}\right) x+\left(\mu_{0}^{2} \sigma_{1}^{2}-\mu_{1}^{2} \sigma_{0}^{2}\right)-\sigma_{0}^{2} \sigma_{1}^{2}\left(\log \sigma_{1}^{2}-\log \sigma_{0}^{2}\right)=0
\end{aligned}
$$

이는 $\sigma_{0}^{2}=\sigma_{1}^{2} \mu_{0} \neq \mu_{1}$ 일 때 $x$ 에 대한 1 차방정식으로 $x=\left(\mu_{0}+\mu_{1}\right) / 2$ 가 유일해가 되고, $\sigma_{0}^{2} \neq \sigma_{1}^{2}$ 일 때는 $x$ 에 대한 이차방정식이 되는데 이의 판별식(Determinant) $D^{\prime}$ 은 다음과 같고 이로부터 서로 다른 두 점 $x=a, x=b$ 에서 만남을 알 수 있다.

$$
\begin{aligned}
D^{\prime} & =\left(\mu_{0} \sigma_{1}^{2}-\mu_{1} \sigma_{0}^{2}\right)^{2}-\left(\sigma_{1}^{2}-\sigma_{0}^{2}\right)\left(\mu_{0}^{2} \sigma_{1}^{2}-\mu_{1}^{2} \sigma_{0}^{2}\right)+\left(\sigma_{1}^{2}-\sigma_{0}^{2}\right) \sigma_{0}^{2} \sigma_{1}^{2}\left(\log \sigma_{1}^{2}-\log \sigma_{0}^{2}\right) \\
& =\sigma_{0}^{2} \sigma_{1}^{2}\left(\mu_{1}-\mu_{0}\right)^{2}+\left(\sigma_{1}^{2}-\sigma_{0}^{2}\right) \sigma_{0}^{2} \sigma_{1}^{2}\left(\log \sigma_{1}^{2}-\log \sigma_{0}^{2}\right) \\
& =\sigma_{0}^{2} \sigma_{1}^{2}\left[\left(\mu_{1}-\mu_{0}\right)^{2}+\left(\sigma_{1}^{2}-\sigma_{0}^{2}\right)\left(\log \sigma_{1}^{2}-\log \sigma_{0}^{2}\right)\right]>0, \text { if } \sigma_{0}^{2} \neq \sigma_{1}^{2}
\end{aligned}
$$

[따름정리2] 두 정규분포 $N\left(\mu_{0}, \sigma_{0}^{2}\right)$ 와 $N\left(\mu_{1}, \sigma_{1}^{2}\right)$ 사이의 분포거리 $D_{1}(0,1)$ 는 다음과 같다.

i) $\mu_{0}=\mu_{1}, \sigma_{0}^{2}=\sigma_{1}^{2}\left(=\sigma^{2}\right)$ 일 때 $D_{1}(0,1)=0$,

ii) $\mu_{0} \neq \mu_{1}, \sigma_{0}^{2}=\sigma_{1}^{2}\left(=\sigma^{2}\right)$ 일 때 $D_{1}(0,1)=2 \Phi\left(\left|\mu_{1}-\mu_{0}\right| / 2 \sigma\right)-1$,

iii) $\sigma_{0}^{2} \neq \sigma_{1}^{2}$ 일 때 $D_{1}(0,1)=\left|\Phi\left(\sigma_{0} \alpha+\sigma_{1} \beta\right)-\Phi\left(\sigma_{0} \alpha-\sigma_{1} \beta\right)-\Phi\left(\sigma_{1} \alpha+\sigma_{0} \beta\right)+\Phi\left(\sigma_{1} \alpha-\sigma_{0} \beta\right)\right|$,

여기서, $\alpha=-\left(\mu_{1}-\mu_{0}\right) /\left(\sigma_{1}^{2}-\sigma_{0}^{2}\right), \quad \beta=\sqrt{\alpha^{2}+\left(\log \sigma_{1}^{2}-\log \sigma_{0}^{2}\right) /\left(\sigma_{1}^{2}-\sigma_{0}^{2}\right)}$ 이고 $\quad$ ( ) 은 표준정 규분포의 분포함수( $c d f)$ 이다.

(증명) 여기에서도 i)은 자명하므로 ii)와 iii)만 증명한다. ii)의 경우 우선 $\mu_{0}<\mu_{1}$ 라고 하자. 여기서 [따름정리1]의 (ii)를 이용하면 


$$
\begin{aligned}
D_{1}(0,1) & =\frac{1}{2} \int_{-\infty}^{\infty}\left|\phi_{0}(x)-\phi_{1}(x)\right| d x \\
& =\frac{1}{2}\left\{\int_{-\infty}^{\left(\mu_{0}+\mu_{1}\right) / 2}\left[\phi_{0}(x)-\phi_{1}(x)\right] d x+\int_{\left(\mu_{0}+\mu_{1}\right) / 2}^{\infty}\left[\phi_{1}(x)-\phi_{0}(x)\right] d x\right\} \\
& =\Phi\left(\frac{\left(\mu_{0}+\mu_{1}\right) / 2-\mu_{0}}{\sigma}\right)-\Phi\left(\frac{\left(\mu_{0}+\mu_{1}\right) / 2-\mu_{1}}{\sigma}\right) \\
& =2 \Phi\left(\frac{\mu_{1}-\mu_{0}}{2 \sigma}\right)-1
\end{aligned}
$$

가 얻어진다. 그리고 $\mu_{0}>\mu_{1}$ 라면 $D_{1}(0,1)=2 \Phi\left(\left(\mu_{0}-\mu_{1}\right) / 2 \sigma\right)-1$ 가 비슷한 과정을 통해 얻어진다. 따라서 ii)가 증명된다.

iii)을 증명하기에 앞서 우선 서로 다른 분산을 가지는 두 정규분포의 확률밀도함수 곡선들 사이 에는 다음의 그래프적 관계가 있음을 [따름정리1]로부터 쉽게 얻을 수 있다([그림2] 참조). 즉, $\mu_{1}$, $\mu_{2}$ 의 크기에 관계없이 $(a, b)$ 구간 밖에서는 분산이 큰 분포의 확률밀도함수 곡선이 분산이 작은 분포의 확률밀도함수 곡선보다 위에 있고, $(a, b)$ 구간 안에서는 반대로 분산이 큰 분포의 확률밀 도함수 곡선이 분산이 작은 분포의 확률밀도함수 곡선보다 아래에 있게 된다. 이제 iii)을 증명하 면 다음과 같다.

$$
\begin{aligned}
D_{1}(0,1) & =\frac{1}{2}\left|\int_{-\infty}^{a}\left[\phi_{1}(x)-\phi_{0}(x)\right] d x+\int_{a}^{b}\left[\phi_{0}(x)-\phi_{1}(x)\right] d x+\int_{b}^{\infty}\left[\phi_{1}(x)-\phi_{0}(x)\right] d x\right| \\
& =\left|\int_{a}^{b} \phi_{0}(x) d x-\int_{a}^{b} \phi_{1}(x) d x\right| \\
& =\left|\Phi\left(\frac{b-\mu_{0}}{\sigma_{0}}\right)-\Phi\left(\frac{a-\mu_{0}}{\sigma_{0}}\right)-\Phi\left(\frac{b-\mu_{1}}{\sigma_{1}}\right)+\Phi\left(\frac{a-\mu_{1}}{\sigma_{1}}\right)\right| \\
& =\left|\Phi\left(\sigma_{0} \alpha+\sigma_{1} \beta\right)-\Phi\left(\sigma_{0} \alpha-\sigma_{1} \beta\right)-\Phi\left(\sigma_{1} \alpha+\sigma_{0} \beta\right)+\Phi\left(\sigma_{1} \alpha-\sigma_{0} \beta\right)\right|
\end{aligned}
$$

\section{4.결합분포간 거리추정량과 우도비 검정통계량의 관계}

만일 $X_{1}, X_{2}, \cdots, X_{n}$ 이 $f(x ; \theta)$ 로부터 얻어진 확률표본이라 한다면 이들의 결합확률밀도함수는 $f(x ; \theta)=f\left(x_{1}, x_{2}, \cdots, x_{n} ; \theta\right)=\Pi_{i=1}^{n} f\left(x_{i} ; \theta\right)$ 가 된다. 그리고 귀무가설과 대립가설을 각각 $H_{0}: \theta \in \omega$, $H_{1}: \theta \in(\Omega-\omega)$ 이라 한다면, 그리고 모수공간 $\omega$ 에서 추정된 $\theta$ 의 추정량을 $\underline{\theta}_{0}$, 모수공간 $\Omega$ 에서 추정된 $\theta$ 의 추정량을 $\hat{\theta}_{1}$ 이라 한다면 $\omega$ 와 $\Omega$ 에서 각각 추정된 결합확률밀도함수들 $f_{0}\left(x ; \hat{\theta}_{0}\right)$, $f_{1}\left(x ; \widehat{\theta}_{1}\right)$ 은 다음과 같다.

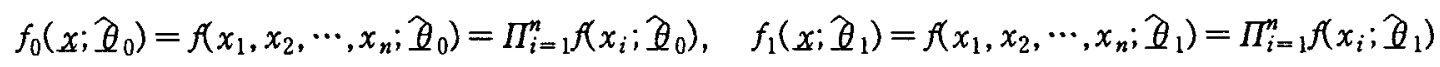

여기서 $f_{0}\left(x ; \hat{\theta}_{0}\right)$ 와 $f_{1}\left(x ; \hat{\theta}_{1}\right)$ 가 우도(likelihood)들이 아니라 결합확률밀도함수들 이라는 점에 유 의할 필요가 있다. 다시 말하면 $\hat{\theta}_{0}, \hat{\theta}_{1}$ 는 추정량들로서 비록 $x$ 의 함수처럼 표현되지만 이들은 
$f_{0}\left(x ; \widehat{\theta}_{0}\right)$ 와 $f_{1}\left(x ; \underline{\theta}_{1}\right)$ 의 변수를 표시하는 $x$ 와 결합되면 안 된다는 것이다. 물론 우도는 이들의 결 합을 통해서 얻어진다. 따라서 아래 [정의2]에서 $\widehat{D}_{n}(0,1)$ 을 계산할 때는 $\underline{\theta}_{0}$ 과 $\hat{\theta}_{1}$ 을 상수 취급 하여야 한다.

[정의2] 모수공간 $\omega$ 에서 추정된 결합확률밀도함수 $f_{0}\left(x: \underline{\theta}_{0}\right)$ 와 모수공간 $\Omega$ 에서 추정된 결합확 률밀도함수 $f_{1}\left(x: \underline{\theta}_{1}\right)$ 사이의 분포간 거리를 '결합분포간 거리추정량'이라 하고, $\widehat{D}_{n}(0,1)$ 로 표현 한다.

$$
\widehat{D}_{n}(0,1)=\frac{1}{2} \int_{R^{n}}\left|f_{0}\left(x ; \widehat{\vartheta}_{0}\right)-f_{1}\left(x ; \underline{\vartheta}_{1}\right)\right|(d x)
$$

사실 결합분포 거리통계량을 정의함에 있어서 $\hat{\theta}_{0}, \hat{\theta}_{1}$ 이 반드시 최대우도법에 의한 추정량일 필요는 없다. 그러나 이들이 최우추정량일 때는 결합분포 거리통계량은 우도비 검정통계량과 밀접 한 관계를 가진다. 이하에서는 정규모집단에서의 모평균과 모분산에 관한 가설검정에서 $\vec{\theta}_{0}, \vec{\theta}_{1}$ 이 최우추정량일 경우의 결합분포 거리통계량이 우도비 검정통계량의 함수가 된다는 것을 몇 가 지의 경우에 대해 보이고자 한다.

참고로, $f(x ; \theta)=\phi\left(x ; \mu, \sigma^{2}\right)$ 이라면 즉 $X_{1}, X_{2}, \cdots, X_{n}$ 이 평균이 $\mu$, 분산이 $\sigma^{2}$ 인 정규분포로부터 얻어진 확률표본이라고 한다면 결합확률밀도함수 $f(x ; \theta)$ 은 $n$ 변량정규분포 $N_{n}\left(\mu 1_{n}, \sigma^{2} I_{n}\right)$ 의 확률 밀도함수 $\phi_{n}\left(\mu 1_{n}, \sigma^{2} I_{n}\right)$ 가 된다는 것은 이하 부분들을 이해하는데 필요한 중요한 사실이 된다.

[ $Z$-검정] $X_{1}, X_{2}, \cdots, X_{n}$ 이 모분산이 $\sigma_{0}^{2}$ 인 것으로 알려진 정규모집단 $N\left(\mu, \sigma_{0}^{2}\right)$ 로부터 얻어진 확률 표본이라 하자. 귀무가설과 대립가설이 $H_{0}: \mu=\mu_{0}, H_{1}: \mu>\mu_{0}$ 인 검정문제에 대한 우도비 검정통계 량은 $z=\sqrt{n}\left(\bar{x}-\mu_{0}\right) / \sigma_{0}$ 이다. 이에 대한 결합분포간 거리추정량을 구해보자. 이 검정문제에서의 결 합확률밀도함수 $f(x ; \theta)$, 모수 $\theta$, 모수공간들 $\omega$ 와 $\Omega, \omega$ 에서 추정된 $\theta$ 와 $f(x ; \theta)$ 의 추정량 $\theta_{0}$ 와 $f_{0}\left(x ; \underline{\theta}_{0}\right), \Omega$ 에서 추정된 $\theta$ 와 $f(x ; \theta)$ 의 추정량 $\underline{\theta}_{1}$ 와 $f_{1}\left(x ; \underline{\theta}_{1}\right)$ 은 각각 다음과 같다.

$$
\begin{aligned}
& f(x ; \underline{\theta})=\phi_{n}\left(\mu 1_{n}, \sigma_{0}^{2} I_{n}\right), \quad \theta=\mu, \quad \omega=\left\{\mu ; \mu=\mu_{0}\right\}, \quad \Omega=\left\{\mu ; \mu \geq \mu_{0}\right\}
\end{aligned}
$$

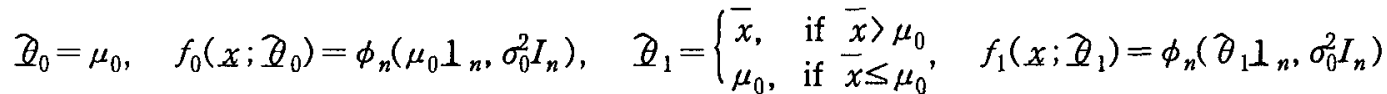

따라서 이 경우는 두 결합분포의 공분산행렬이 동일한 경우이므로 [정리2]의 iii)을 이용하여 결 합분포간 거리추정량을 구하기 위해서는 [정리2]의 $\mathrm{iii}$ )의 조건에 의해 $\bar{x}>\mu_{0}$ 인 경우에는 $\mu_{a}=\mu_{0} 1_{n}, \quad \Sigma_{a}=\sigma_{0}^{2} I_{n}, \quad \mu_{b}=\bar{x} 1_{n}, \quad \Sigma_{b}=\sigma_{0}^{2} I_{n}$ (즉, $\quad v=\bar{x}, \quad u=\mu_{0}, \quad k=\sigma_{0}$ )라고, $\bar{x} \leq \mu_{0}$ 인 경우에는 $\mu_{a}=\mu_{0} 1_{n}, \quad \Sigma_{a}=\sigma_{0}^{2} I_{n}, \quad \mu_{b}=\mu_{0} 1_{n}, \quad \Sigma_{b}=\sigma_{0}^{2} I_{n}$ (즉, $\quad v=\mu_{0}, \quad u=\mu_{0}, \quad k=\sigma_{0}$ )라고 두어야 한다. 따라서 결합분포간 거리추정량은 다음과 같다. 


$$
\widehat{D}_{n}(0,1)= \begin{cases}2 \Phi\left(\sqrt{n}\left(\bar{x}-\mu_{0}\right) / 2 \sigma_{0}\right)-1, & \bar{x}>\mu_{0} \text { 일 때 } \\ 0, & x \leq \mu_{0} \text { 일 때 }\end{cases}
$$

그런데 이는 우도비 검정통계량인 $z=\sqrt{n}\left(\bar{x}-\mu_{0}\right) / \sigma_{0}$ 의 함수인 것을 볼 수 있으며, $z$ 의 값이 클 수록. $\widehat{D}_{n}(0,1)$ 의 값도 커짐을 알 수 있다.

[ $\boldsymbol{T}$-검정(단일집단)] 확률표본 $X_{1}, X_{2}, \cdots, X_{n}$ 이 $\mu$ 와 $\sigma^{2}$ 가 미지인 정규모집단 $N\left(\mu, \sigma^{2}\right)$ 로부터 얻 어진 것으로 가정하자. 귀무가설과 대립가설이 $H_{0}: \mu=\mu_{0}, H_{1}: \mu \neq \mu_{0}$ 인 검정문제에 대한 우도비 검 정통계량은 $t=\sqrt{n}\left(\bar{x}-\mu_{0}\right) / s_{*}$ 인 것으로 널리 알려져 있다. 여기서, $s_{*}^{2}=\sum_{i=1}^{n}\left(x_{i}-\bar{x}\right)^{2} /(n-1)$ 이다. 이 문제에 대한 결합분포간 거리추정량도 구해보자. 이 검정문제에서의 결합확률밀도함수 $f(x ; \theta)$, 모수 $\theta$, 모수공간들 $\omega$ 와 $\Omega, \omega$ 에서 추정된 $\theta$ 와 $f(x ; \theta)$ 의 추정량 $\underline{\underline{\theta}}_{0}$ 와 $f_{0}\left(x ; \underline{\underline{\theta}}_{0}\right), \Omega$ 에서 추정 된 $\underline{\theta}$ 와 $f(x ; \theta)$ 의 추정량 $\hat{\theta}_{1}$ 와 $f_{1}\left(x ; \hat{\theta}_{1}\right)$ 은 각각 다음과 같다.

$$
\begin{aligned}
& f(x ; \theta)=\phi_{n}\left(\mu 1_{n}, \sigma^{2} I_{n}\right), \quad \theta=\left(\mu \sigma^{2}\right)^{\prime}, \\
& \omega=\left\{\left(\mu \sigma^{2}\right)^{\prime} ; \mu=\mu_{0}, 0<\sigma^{2}<\infty\right\}, \quad \Omega=\left\{\left(\mu \sigma^{2}\right)^{\prime} ;-\infty<\mu<\infty, 0<\sigma^{2}<\infty\right\}, \\
& \widehat{\vartheta}_{0}=\left(\mu_{0} s_{0}^{2}\right)^{\prime}, \quad f_{0}\left(x ; \widehat{\theta}_{0}\right)=\phi_{n}\left(\mu_{0} 1_{n}, s_{0}^{2} I_{n}\right), \quad \widehat{\theta}_{1}=\left(\bar{x} s^{2}\right)^{\prime}, \quad f_{1}\left(x ; \widehat{\vartheta}_{1}\right)=\phi_{n}\left(\bar{x} 1_{n}, s^{2} I_{n}\right)
\end{aligned}
$$

여기서 $s_{0}^{2}=\sum_{i=1}^{n}\left(x_{i}-\mu_{0}\right)^{2} / n, \quad s^{2}=\sum_{i=1}^{n}\left(x_{i}-\bar{x}\right)^{2} / n$ 으로 $\quad s_{0}^{2}=s^{2}+\left(\bar{x}-\mu_{0}\right)^{2}, \quad s^{2}=(n-1) s_{*}^{2} / n$ 라는 관계가 있다. [정리3]의 iv)에 의해 결합분포간 거리추정량을 구하기 위해서는 $\mu_{a}=\mu_{0} 1_{n}$, $\left.\Sigma_{a}=s_{0}^{2} I_{n}, \quad \mu_{b}=\bar{x}\right]_{n}, \quad \Sigma_{b}=s^{2} I_{n}$ 라고 두어야 한다. 왜냐하면 [정리3]의 결과를 이용하기 위해서는 $\left(\Sigma_{a}-\Sigma_{b}\right)$ 가 양정치행렬이 되어야 하기 때문이다. 그러면 우선 다음과 같은 관계들을 얻을 수 있 는데 이의 중간 계산과정은 별첨의 (I)부분에 남긴다.

$$
\begin{gathered}
\Sigma_{a}^{1 / 2} \Sigma_{b}^{-1} \Sigma_{a}^{1 / 2}-I=\left\{t^{2} /(n-1)\right\} I_{n}, \quad I-\Sigma_{b}^{1 / 2} \Sigma_{a}^{-1} \Sigma_{b}^{1 / 2}=\left\{t^{2} /(n-1)\right\} /\left\{1+t^{2} /(n-1)\right\} I_{n}, \\
\eta_{a}=\left(\sqrt{n+n\left\{t^{2} /(n-1)\right\}^{-1}} 0 \cdots 0\right)^{\prime}, \quad \eta_{b}=\left(\sqrt{n\left\{t^{2} /(n-1)\right\}^{-1}} 0 \cdots 0\right)^{\prime}, \quad \beta=n+n \log \left(1+t^{2} /(n-1)\right)
\end{gathered}
$$

따라서 [정리3]-vi와 [성질3]으로부터 다음과 같은 결합분포간 거리추정량 $\widehat{D}_{n}(0,1)$ 을 얻을 수 있는데 이는 완전히 $t^{2}=n\left(\bar{x}-\mu_{0}\right)^{2} / s_{*}^{2}$ 의 함수로서 $\sigma^{2}$ 에 의존하지 않음을 알 수 있다.

$$
\begin{aligned}
& \widehat{D}_{n}(0,1)=\Phi_{n}\left(\left(\sqrt{n+n\left\{t^{2} /(n-1)\right\}^{-1}} 0 \cdots 0\right)^{\prime}, I_{n},\left\{n+n \log \left(1+t^{2} /(n-1)\right)\right\}\left\{t^{2} /(n-1)\right\}^{-1}\right) \\
& -\Phi_{n}\left(\left(\sqrt{n\left\{t^{2} /(n-1)\right\}^{-1}} \quad 0 \cdots 0\right)^{\prime}, I_{n},\left\{n+n \log \left(1+t^{2} /(n-1)\right)\right\}\left\{1+\left\{t^{2} /(n-1)\right\}^{-1}\right\}\right)
\end{aligned}
$$


[ $\chi^{2}$-검정] 확률표본 $X_{1}, X_{2}, \cdots, X_{n}$ 이 $\mu$ 와 $\sigma^{2}$ 가 미지인 정규모집단 $N\left(\mu, \sigma^{2}\right)$ 로부터 얻어진 것으 로 가정하자. 귀무가설과 대립가설이 $H_{0}: \sigma^{2}=\sigma_{0}^{2}, H_{1}: \sigma^{2} \neq \sigma_{0}^{2}$ 인 검정문제에 대한 일반우도비 검정통 계량은 $\chi^{2}=n s^{2} / \sigma_{0}^{2}$ 인 것으로 널리 알려져 있다. 이에 대한 결합분포간 거리추정량을 구해보자. 이 검정문제에서의 결합확률밀도함수 $f(x ; \theta)$, 모수 $\theta$, 모수공간들 $\omega$ 와 $\Omega, \omega$ 에서 추정된 $\theta$ 와 $f(x ; \theta)$ 의 추정량 $\underline{\theta}_{0}$ 와 $f_{0}\left(x ; \underline{\theta}_{0}\right), \Omega$ 에서 추정된 $\theta$ 와 $f(x ; \theta)$ 의 추정량 $\underline{\theta}_{1}$ 와 $f_{1}\left(x ; \hat{\theta}_{1}\right)$ 은 각각 다음과 같다.

$$
\begin{gathered}
f(x ; \theta)=\phi_{n}\left(\mu 1_{n}, \sigma^{2} I_{n}\right), \quad \theta=\left(\mu \sigma^{2}\right)^{\prime}, \\
\omega=\left\{\left(\mu \sigma^{2}\right)^{\prime}:-\infty<\mu<\infty, \sigma^{2}=\sigma_{0}^{2}\right\}, \quad \Omega=\left\{\left(\mu \sigma^{2}\right)^{\prime}:-\infty<\mu<\infty, 0<\sigma^{2}<\infty\right\}, \\
\underline{\theta}_{0}=\left(\bar{x} \sigma_{0}^{2}\right)^{\prime}, \quad f_{0}\left(x ; \underline{\theta}_{0}\right)=\phi_{n}\left(\bar{x} 1_{n}, \sigma_{0}^{2} I_{n}\right), \quad \underline{\theta}_{1}=\left(\begin{array}{ll}
\bar{x} & s^{2}
\end{array}\right)^{\prime}, \quad f_{1}\left(x ; \underline{\theta}_{1}\right)=\phi_{n}\left(\bar{x} 1_{n}, s^{2} I_{n}\right)
\end{gathered}
$$

[정리3]의 iv)에 의해 결합분포간 거리추정량을 구하기 위해서는 $\sigma_{0}^{2}>s^{2}$ 인 경우는 $\Perp_{a}=\bar{x} 1_{n}$, $\Sigma_{a}=\sigma_{0}^{2} I_{n}, \quad \mu_{b}=\bar{x} 1_{n}, \quad \Sigma_{b}=s^{2} I_{n}$ 라고 두어야 한다. 그리고 $\sigma_{0}^{2}<s^{2}$ 인 경우는 $\mu_{a}=\bar{x} 1_{n}, \quad \Sigma_{a}=s^{2} I_{n}$, $\mu_{b}=\bar{x} 1_{n}, \quad \Sigma_{b}=\sigma_{0}^{2} I_{n}$ 라고 두어야 한다. 왜냐하면 [정리3]의 결과를 이용하기 위해서는 $\left(\Sigma_{a}-\Sigma_{b}\right)$ 가 양정치행렬이 되어야 하기 때문이다. 그러면 $\sigma_{0}^{2}>s^{2}$ 인 경우 우선 다음과 같은 관계들을 얻을 수 있는데 이의 중간 계산과정은 별첨의 (II)-1 부분에 남긴다.

$$
\begin{aligned}
\Sigma_{a}^{1 / 2} \Sigma_{b}^{-1} \Sigma_{a}^{1 / 2}-I= & \left(n / \chi^{2}-1\right) I_{n}, I-\Sigma_{b}^{1 / 2} \Sigma_{a}^{-1} \Sigma_{b}^{1 / 2}=\left(1-\chi^{2} / n\right) I_{n}, \quad \eta_{a}=0, \quad \eta_{b}=0, \quad \beta=n \log \left(n / \chi^{2}\right) \\
D_{n}(0,1) & =\Phi_{n}\left(0,\left(n / \chi^{2}-1\right) I_{n}, n \log \left(n / \chi^{2}\right)\right)-\Phi_{n}\left(0,\left(1-\chi^{2} / n\right) I_{n}, n \log \left(n / \chi^{2}\right)\right) \\
& =\Phi_{n}\left(0, I_{n},\left\{n \log \left(n / \chi^{2}\right)\right\} /\left(n / \chi^{2}-1\right)\right)-\Phi_{n}\left(0, I_{n},\left\{n \log \left(n / \chi^{2}\right)\right\} /\left(1-\chi^{2} / n\right)\right)
\end{aligned}
$$

그리고 $\sigma_{0}^{2}<s^{2}$ 인 경우는 다음과 같은 관계들을 얻을 수 있는데 이의 중간 계산과정은 별첨의 (II) -2 부분에 남긴다.

$$
\begin{aligned}
\Sigma_{a}^{1 / 2} \Sigma_{b}^{-1} \Sigma_{a}^{1 / 2}-I & =\left(\chi^{2} / n-1\right) I_{n}, I-\Sigma_{b}^{1 / 2} \Sigma_{a}^{-1} \Sigma_{b}^{1 / 2}=\left(1-n / \chi^{2}\right) I_{n}, \quad \eta_{a}=0, \quad \eta_{b}=0, \quad \beta=n \log \left(\chi^{2} / n\right) \\
\widehat{D}_{n}(0,1) & =\Phi_{n}\left(0,\left(\chi^{2} / n-1\right) I_{n}, n \log \left(\chi^{2} / n\right)\right)-\Phi_{n}\left(0,\left(1-n / \chi^{2}\right) I_{n}, n \log \left(\chi^{2} / n\right)\right) \\
& =\Phi_{n}\left(0, I_{n},\left\{n \log \left(\chi^{2} / n\right)\right\} /\left(\chi^{2} / n-1\right)\right)-\Phi_{n}\left(0, I_{n},\left\{n \log \left(\chi^{2} / n\right)\right\} /\left(1-n / \chi^{2}\right)\right)
\end{aligned}
$$

[ $T$-검정(두 독립집단)] 독립인 두 정규모집단 $N\left(\mu_{1}, \sigma^{2}\right), N\left(\mu_{2}, \sigma^{2}\right)$ 으로부터 표본의 크기가 $n_{1}$, $n_{2}$ 인 확률표본을 얻었다고 가정하자. 여기서 공통 모분산인 $\sigma^{2}$ 은 미지이다. 이 때 귀무가설과 대 립가설이 $H_{0}: \mu_{1}=\mu_{2}, \quad H_{1}: \mu_{1} \neq \mu_{2}$ 인 검정문제에 대한 우도비 검정통계량은 $t_{p}=\left(\bar{x}_{1}-\bar{x}_{2}\right) /\left\{s_{p *} \sqrt{1 / n_{1}+1 / n_{2}}\right\}$ 로서, $s_{p *}^{2}=\sum_{i=1}^{Q} \Sigma_{j-1}^{n_{i}}\left(x_{i j}-\bar{x}_{i}\right)^{2} /(n-2)$ 이다. 이 문제에 대한 결합분 포간 거리추정량을 구해보자. 이 검정문제에서의 결합확률밀도함수 $f(x ; \theta)$, 모수 $\theta$, 모수공간들 
$\omega$ 와 $\Omega, \omega$ 에서 추정된 $\underline{\theta}$ 와 $f(x ; \theta)$ 의 추정량 $\widehat{\theta}_{0}$ 와 $f_{0}\left(x ; \underline{\theta}_{0}\right), \Omega$ 에서 추정된 $\theta$ 와 $f(x ; \theta)$ 의 추 정량 $\hat{\underline{\theta}}_{1}$ 와 $f_{1}\left(x ; \hat{\theta}_{1}\right)$ 은 각각 다음과 같다.

$$
\begin{aligned}
& f(x ; \theta)=\phi_{n_{1}}\left(x_{1} ; \mu_{1} 1_{n_{1}}, \sigma^{2} I_{n_{1}}\right) \cdot \phi_{n_{2}}\left(x_{2} ; \mu_{2} 1_{n_{2}}, \sigma^{2} I_{n_{2}}\right)=\phi_{n}(x ; \underline{\mu}, \Sigma), \quad \theta=\left(\mu_{1} \mu_{2} \sigma^{2}\right)^{\prime} \\
& \omega=\left\{\left(\begin{array}{lll}
\mu_{1} & \mu_{2} & \sigma^{2}
\end{array}\right)^{\prime} ;-\infty<\mu_{1}=\mu_{2}<\infty, 0<\sigma^{2}<\infty\right\}, \Omega=\left\{\left(\mu_{1} \mu_{2} \sigma^{2}\right)^{\prime} ;-\infty<\mu_{1}<\infty,-\infty<\mu_{2}<\infty, 0<\sigma^{2}<\infty\right\} \\
& \underline{\theta}_{0}=\left(\bar{m} \bar{m} s_{m}^{2}\right)^{\prime}, \quad f_{0}\left(\underline{x} ; \underline{\theta}_{0}\right)=\phi_{n}\left(x ; \bar{m} 1_{n}, s_{m}^{2} I_{n}\right), \quad \underline{\underline{\theta}}_{1}=\left(\bar{x}_{1} \bar{x}_{2} s_{p}^{2}\right)^{\prime}, f_{1}\left(x ; \underline{\underline{t}}_{1}\right)=\phi_{n}\left(\underline{x} ; \bar{x}, s_{p}^{2} I_{n}\right)
\end{aligned}
$$

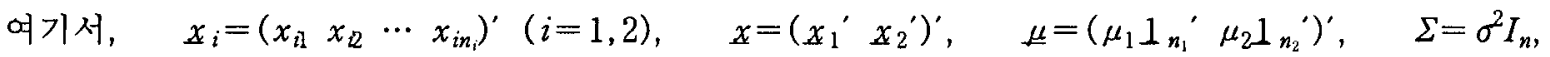

$$
\begin{aligned}
& n=n_{1}+n_{2}, \quad \bar{x}_{i}=\sum_{j=1}^{n_{i}} x_{i j} / n_{i}(i=1,2), \quad \bar{m}=\left(n_{1} \bar{x}_{1}+n_{2} \bar{x}_{2}\right) / n, \quad \bar{x}=\left(\bar{x}_{1} 1_{n_{1}}{ }^{\prime} \bar{x}_{2} \perp_{n_{2}}{ }^{\prime}\right)^{\prime} \text {, } \\
& s_{m}^{2}=\sum_{i=1}^{2} \Sigma_{j=1}^{n_{1}}\left(x_{i j}-\bar{m}\right)^{2} / n, \quad s_{p}^{2}=\sum_{i=1}^{2} \sum_{j=1}^{n_{1}}\left(x_{i j}-\bar{x}_{i}\right)^{2} / n \text { 으로, } \quad s_{m}^{2}=s_{p}^{2}+\left(n_{1} n_{2} / n^{2}\right)\left(\bar{x}_{1}-\bar{x}_{2}\right)^{2} \text {, }
\end{aligned}
$$

[정리3]의 iv)에 의해 결합분포간 거리추정량을 구하기 위해서는 $\underline{\mu}_{a}=\bar{m} 1_{n}, \Sigma_{a}=s_{m}^{2} I_{n}, \underline{\mu}_{b}=\bar{x}$, $\Sigma_{b}=s_{p}^{2} I_{n}$ 이라고 두어야 한다. 왜냐하면 [정리3]의 결과를 이용하기 위해서는 $\left(\Sigma_{a}-\Sigma_{b}\right)$ 가 양정치행 렬이 되어야 하기 때문이다. 그러면 우선 다음과 같은 관계들을 얻을 수 있는데 이의 중간 계산과 정은 별첨의 (III)부분에 남긴다.

$$
\begin{aligned}
& \Sigma_{a}^{1 / 2} \Sigma_{b}^{-1} \Sigma_{a}^{1 / 2}-I=t_{p}^{2} /(n-2) I_{n}, \quad I-\Sigma_{b}^{1 / 2} \Sigma_{a}^{-1} \Sigma_{b}^{1 / 2}=\left\{t_{p}^{2} /(n-2)\right\} /\left\{1+t_{p}^{2} /(n-2)\right\} I_{n}, \\
& \underline{\eta}_{a}=\left(\sqrt{n+n\left(t_{p}^{2} /(n-2)\right)^{-1}} 0 \cdots 0\right)^{\prime}, \quad \eta_{b}=\left(\sqrt{n\left(t_{p}^{2} /(n-2)\right)^{-1}} 0 \cdots\right)^{\prime}, \quad \beta=n+n \log \left(1+t_{p}^{2} /(n-2)\right) \\
& \widehat{D}_{n}(0,1)=\Phi_{n}\left(\left(\sqrt{n+n\left(t_{p}^{2} /(n-2)\right)^{-1}} 0 \cdots 0\right)^{\prime}, I_{n},\left\{n+n \log \left(1+t_{p}^{2} /(n-2)\right)\right\}\left\{t_{p}^{2} /(n-2)\right\}^{-1}\right) \\
& -\Phi_{n}\left(\left(\sqrt{n\left(t_{p}^{2} /(n-2)\right)^{-1}} \quad 0 \cdots 0\right)^{\prime}, I_{n},\left\{n+n \log \left(1+t_{p}^{2} /(n-2)\right)\right\}\left\{1+\left\{t_{p}^{2} /(n-2)\right\}^{-1}\right\}\right)
\end{aligned}
$$

이로부터 $\widehat{D}_{n}(0,1)$ 은 모두 $t_{p}^{2}$ 에만 의존함을 볼 수 있다

[일원분산분석] 독립인 $k$ 개의 정규모집단 $N\left(\mu_{i}, \sigma^{2}\right)(i=1,2, \cdots, k)$ 으로부터 각각 표본의 크기가 $n_{i}$ 인 확률표본들을 얻었다고 가정하자. 여기서 공통 모분산인 $\sigma^{2}$ 은 미지이다. 이 때 귀무가설과 대립가설이 $H_{0}: \mu_{1}=\mu_{2}=\cdots=\mu_{k}, H_{1}: n o t H_{0}$ 인 일원분산분석 문제에 대한 우도비 검정통계량은 $F=\left\{n\left(s_{m}^{2}-s_{p}^{2}\right) /(k-1)\right\} /\left\{n s_{p}^{2} /(n-k)\right\}$ 인 것으로 잘 알려져 있다. 여기서 표현들의 정의는 아래를 참조하면 된다. 이 문제에 대한 결합분포간 거리추정량을 구해보자. 이 검정문제에서의 결합확률 밀도함수 $f(x ; \theta)$, 모수 $\theta$, 모수공간들 $\omega$ 와 $\Omega, \omega$ 에서 추정된 $\theta$ 와 $f(x ; \theta)$ 의 추정량 $\hat{\theta}_{0}$ 와 $f_{0}\left(x ; \hat{\theta}_{0}\right), \Omega$ 에서 추정된 $\theta$ 와 $\left.R x ; \theta\right)$ 의 추정량 $\hat{\theta}_{1}$ 와 $f_{1}\left(x ; \hat{\theta}_{1}\right)$ 은 각각 다음과 같다.

$$
\begin{aligned}
& f(\underline{x})=\Pi_{i=1}^{k} \phi_{n_{i}}\left(x_{i} ; \mu_{i} I_{n_{i}}, \sigma^{2} I_{n_{i}}\right)=\phi_{n}(\underline{x} ; \underline{\mu}, \Sigma), \quad \underline{\theta}=\left(\begin{array}{lllll}
\mu_{1} & \mu_{2} & \cdots & \mu_{k} & \sigma^{2}
\end{array}\right)^{\prime}, \\
& \omega=\left\{\left(\begin{array}{lllll}
\mu_{1} & \mu_{2} & \cdots & \mu_{k} & \sigma^{2}
\end{array}\right)^{\prime} ;-\infty<\mu_{1}=\mu_{2}=\cdots=\mu_{k}<\infty, 0<\sigma^{2}<\infty\right\}, \\
& \Omega=\left\{\left(\begin{array}{lllll}
\mu_{1} & \mu_{2} & \cdots & \mu_{k} & \sigma^{2}
\end{array}\right)^{\prime} ;-\infty<\mu_{i}<\infty, i=1,2, \cdots, k, 0<\sigma^{2}<\infty\right\} \\
& \widehat{\theta}_{0}=\left(\bar{m} \bar{m} \cdots \bar{m} s_{m}^{2}\right)^{\prime}, f_{0}\left(x ; \hat{\theta}_{0}\right)=\phi_{n}\left(x ; \bar{m} 1_{n}, s_{m}^{2} I_{n}\right), \\
& \hat{\theta}_{1}=\left(\bar{x}_{1} \bar{x}_{2} \cdots \bar{x}_{k} s_{p}^{2}\right)^{\prime}, f_{1}\left(x ; \hat{\theta}_{1}\right)=\phi_{n}\left(x ; \bar{x}, s_{p}^{2} I_{n}\right) \\
& \text { 여기서, } \quad n=\sum_{i=1}^{k} n_{i}, \quad x_{i}=\left(\begin{array}{llll}
x_{i 1} & x_{i 2} & \cdots & x_{i n_{i}}
\end{array}\right)^{\prime}(i=1,2, \cdots, k), \quad x=\left(\begin{array}{llll}
x_{1}^{\prime} & x_{2}^{\prime} & \cdots & x_{k}^{\prime}
\end{array}\right)^{\prime} \text {, }
\end{aligned}
$$


$\mu=\left(\mu_{1} 1_{n_{1}}{ }^{\prime} \mu_{2} 1_{n_{i}}{ }^{\prime} \cdots \mu_{k} 1_{n_{k}}\right)^{\prime}, \quad \Sigma=\sigma^{2} I_{n}, \quad \bar{x}_{i}=\sum_{j=1}^{n_{i}} x_{i j} / n_{i}(i=1,2, \cdots, k), \quad \bar{m}=\sum_{i=1}^{k} n_{i} \bar{x}_{i} / n$,

$\bar{x}=\left(\bar{x}_{1} 1_{n_{1}}{ }^{\prime} \bar{x}_{2} 1_{n_{2}}{ }^{\prime} \cdots \bar{x}_{k} 1_{n_{k}}^{\prime}\right)^{\prime}, \quad s_{m}^{2}=\sum_{i=1}^{k} \sum_{j=1}^{n_{i}}\left(x_{i j}-\bar{m}\right)^{2} / n, \quad s_{p}^{2}=\sum_{i=1}^{k} \sum_{j=1}^{n_{i}}\left(x_{i j}-\bar{x}_{i}\right)^{2} / n$ 으로, $s_{m}^{2}=s_{p}^{2}+\sum_{i=1}^{k} n_{i}\left(\bar{x}_{i}-\bar{m}\right)^{2} / n$ 의 관계가 있다.

[정리3]의 iv)에 의해 결합분포간 거리추정량을 구하기 위해서는 $\mu_{a}=\bar{m} 1_{n}, \Sigma_{a}=s_{m}^{2} I_{n}, \mu_{b}=\bar{x}$, $\Sigma_{b}=s_{p}^{2} I_{n}$ 이라고 두어야 한다. 왜냐하면 [정리3]의 결과를 이용하기 위해서는 $\left(\Sigma_{a}-\Sigma_{b}\right)$ 가 양정치행 렬이 되어야 하기 때문이다. 그러면 우선 다음과 같은 관계들을 얻을 수 있는데 이의 중간 계산과 정은 별첨의 (IV)부분에 남긴다.

$$
\begin{aligned}
& \Sigma_{a}^{1 / 2} \Sigma_{b}^{-1} \Sigma_{a}^{1 / 2}-I=(k-1) F /(n-k) I_{n}, \quad I-\Sigma_{b}^{1 / 2} \Sigma_{a}^{-1} \Sigma_{b}^{1 / 2}=\{(k-1) F /(n-k)\} /\{(k-1) F /(n-k)+1\} I_{n} \\
& \eta_{a}=\left(\sqrt{n+n\{(k-1) F /(n-k)\}^{-1}} 0 \cdots\right)^{\prime}, \quad \eta_{b}=\left(\sqrt{n\{(k-1) F /(n-k)\}^{-1}} 0 \cdots\right)^{\prime}, \\
& \beta=n+n \log \{1+(k-1) F /(n-k)\} \\
& \left.\widehat{D}_{n}(0,1)=\Phi_{n}\left(\sqrt{n+n\left\{\frac{(k-1) F}{(n-k)}\right\}^{-1}} 0 \cdots 0\right)^{\prime}, I_{n},\left\{n+n \log \left(1+\frac{(k-1) F}{(n-k)}\right)\right\}\left\{\frac{(k-1) F}{(n-k)}\right\}^{-1}\right) \\
& \left.-\Phi_{n}\left(\sqrt{n\left\{\frac{(k-1) F}{(n-k)}\right\}^{-1}} \quad 0 \cdots\right)^{\prime}, I_{n},\left\{n+n \log \left(1+\frac{(k-1) F}{(n-k)}\right)\right\}\left\{1+\left\{\frac{(k-1) F}{(n-k)}\right\}^{-1}\right\}\right)
\end{aligned}
$$

이로부터 $\widehat{D}_{n}(0,1)$ 은 $F$ 의 함수임을 알 수 있다. 물론 이 식에서 $k$ 의 값을 2 로 두고 $T^{2}(d f=v)$ 통계량은 $F(d f=(1, v))$ 통계량과 일치한다는 사실을 이용하면 두 독립집단의 $T$-검정 결과와 일치함을 볼 수 있다.

\section{참고문헌}

[1] Alexander M. Mood, Franklin A. Graybill, Duane C. Boes. (1974). Introduction to the Theory of Statistics, 3rd Edition. McGRAW-Hill.

[2] Robb J. Muirhead. (1982). Aspects of Multivariate Statistical Theory. John Wiley \& Sons, INC. 
[별첨I]

$$
\begin{aligned}
& \Sigma_{a}^{1 / 2} \Sigma_{b}^{-1} \Sigma_{a}^{1 / 2}-I=s_{0} I_{n} \frac{1}{s^{2}} I_{n} s_{0} I_{n}-I_{n}=\frac{s_{0}^{2}-s^{2}}{s^{2}} I_{n}=\frac{\left(\bar{x}-\mu_{0}\right)^{2}}{s^{2}} I_{n}=\frac{\left(\bar{x}-\mu_{0}\right)^{2}}{(n-1) s_{*}^{2} / n} I_{n}=\frac{t^{2}}{(n-1)} I_{n} \\
& I-\Sigma_{b}^{1 / 2} \Sigma_{a}^{-1} \Sigma_{b}^{1 / 2}=I-s I_{n} \frac{1}{s_{0}^{2}} I_{n} s I_{n}=\frac{\left(s_{0}^{2}-s^{2}\right)}{s_{0}^{2}} I_{n}=\frac{\left(\bar{x}-\mu_{0}\right)^{2} / s^{2}}{1+\left(\bar{x}-\mu_{0}\right)^{2} / s^{2}} I_{n}=\frac{\left(t^{2} /(n-1)\right)}{1+\left(t^{2} /(n-1)\right)} I_{n} \\
& \eta_{a}=\left(\sqrt{\left(\underline{\mu}_{b}-\mu_{a}\right)^{\prime}\left(I-\Sigma_{b} \Sigma_{a}^{-1}\right)^{-1} \Sigma_{a}^{-1}\left(I-\Sigma_{b} \Sigma_{a}^{-1}\right)^{-1}\left(\mu_{b}-\mu_{a}\right)} \quad 0 \cdots 0\right)^{\prime} \text { 에서 } \\
& \left(\underline{\mu}_{b}-\underline{\mu}_{a}\right)^{\prime}\left(I-\Sigma_{b} \Sigma_{a}^{-1}\right)^{-1} \Sigma_{a}^{-1}\left(I-\Sigma_{b} \Sigma_{a}^{-1}\right)^{-1}\left(\underline{\mu}_{b}-\mu_{a}\right) \\
& =\left(\bar{x} \perp_{n}-\mu_{0} I_{n}\right)^{\prime}\left(I-s^{2} I_{n} \cdot s_{0}^{-2} I_{n}\right)^{-1} \cdot s_{0}^{-2} I_{n} \cdot\left(I-s^{2} I_{n} \cdot s_{0}^{-2} I_{n}\right)^{-1} \cdot\left(\bar{x} 1_{n}-\mu_{0} 1_{n}\right) \\
& =\left(\bar{x}-\mu_{0}\right)^{2}\left(1-s^{2} s_{0}^{-2}\right)^{-2} s_{0}^{-2} 1_{n}^{\prime} 1_{n}=n\left(\bar{x}-\mu_{0}\right)^{2}\left(1-s^{2} s_{0}^{-2}\right)^{-2} s_{0}^{-2} \\
& =n\left(\bar{x}-\mu_{0}\right)^{2} s_{0}^{2} /\left(s_{0}^{2}-s^{2}\right)^{2}=n s_{0}^{2} /\left(s_{0}^{2}-s^{2}\right)=n\left[1+\left(t^{2} /(n-1)\right)^{-1}\right]
\end{aligned}
$$

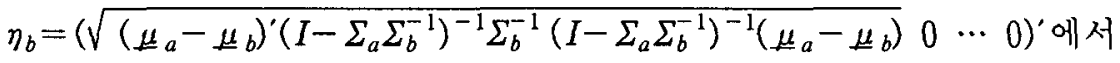

$$
\begin{aligned}
& \left(\underline{\mu}_{a}-\underline{\mu}_{b}\right)^{\prime}\left(I-\Sigma_{a} \Sigma_{b}^{-1}\right)^{-1} \Sigma_{b}^{-1}\left(I-\Sigma_{a} \Sigma_{b}^{-1}\right)^{-1}\left(\underline{\mu}_{a}-\underline{\mu}_{b}\right) \\
& =\left(\mu_{0} 1_{n}-\bar{x} 1_{n}\right)^{\prime}\left(I-s_{0}^{2} I_{n} \cdot s^{-2} I_{n}\right)^{-1} \cdot s^{-2} I_{n} \cdot\left(I-s_{0}^{2} I_{n} \cdot s^{-2} I_{n}\right)^{-1} \cdot\left(\mu_{0} 1_{n}-\bar{x} 1_{n}\right) \\
& =\left(\mu_{0}-\bar{x}\right)^{2}\left(1-s_{0}^{2} s^{-2}\right)^{-2} s^{-2} 1_{n}^{\prime} 1_{n}=n\left(\mu_{0}-\bar{x}\right)^{2}\left(1-s_{0}^{2} s^{-2}\right)^{-2} s^{-2} \\
& =n\left(\mu_{0}-\bar{x}\right)^{2} s^{2} /\left(s^{2}-s_{0}^{2}\right)^{2}=n s^{2} /\left(s_{0}^{2}-s^{2}\right)=n s^{2} /\left(\bar{x}-\mu_{0}\right)^{2}=n\left[t^{2} /(n-1)\right]^{-1} \\
& \beta=\left(\underline{\mu}_{b}-\underline{\mu}_{a}\right)^{\prime}\left(\Sigma_{a}-\Sigma_{b}\right)^{-1}\left(\underline{\mu}_{b}-\underline{\mu}_{a}\right)+\left(\log \left|\Sigma_{a}\right|-\log \left|\Sigma_{b}\right|\right) \\
& =\left(\bar{x} \underline{1}_{n}-\mu_{0} 1_{n}\right)^{\prime}\left(s_{0}^{2} \underline{I}_{n}-s^{2} \underline{I}_{n}\right)^{-1}\left(\bar{x} \underline{1}_{n}-\mu_{0} 1_{n}\right)+\left(\log \left|s_{0}^{2} I_{n}\right|-\log \left|s^{2} I_{n}\right|\right) \\
& =n\left(\bar{x}-\mu_{0}\right)^{2} /\left(s_{0}^{2}-s^{2}\right)+n \log \left(s_{0}^{2} / s^{2}\right)=n+n \log \left\{1+\left(\bar{x}-\mu_{0}\right)^{2} / s^{2}\right\}=n+n \log \left(1+t^{2} /(n-1)\right)
\end{aligned}
$$

\section{[별첨II]-1}

$$
\begin{aligned}
& \Sigma_{a}^{1 / 2} \Sigma_{b}^{-1} \Sigma_{a}^{1 / 2}-I=\sigma_{0} I_{n} \cdot s^{-2} I_{n} \cdot \sigma_{0} I_{n}-I_{n}=\left(\sigma_{0}^{2}-s^{2}\right) / s^{2} \cdot I_{n}=\left(n / \chi^{2}-1\right) I_{n} \\
& I-\Sigma_{b}^{1 / 2} \Sigma_{a}^{-1} \Sigma_{b}^{1 / 2}=I-s I_{n} \cdot \sigma_{0}^{-2} I_{n} \cdot s I_{n}=\left(\sigma_{0}^{2}-s^{2}\right) / \sigma_{0}^{2} \cdot I_{n}=\left(1-\chi^{2} / n\right) I_{n} \\
& \underline{\eta}_{a}=\left(\sqrt{\left(\mu_{b}-\underline{\mu}_{a}\right)^{\prime}\left(I-\Sigma_{b} \Sigma_{a}^{-1}\right)^{-1} \Sigma_{a}^{-1}\left(I-\Sigma_{b} \Sigma_{a}^{-1}\right)^{-1}\left(\underline{\mu}_{b}-\underline{\mu}_{a}\right)} 0 \cdots 0\right)^{\prime} \text { 에서 } \\
& \underline{\mu}_{a}=\underline{\mu}_{b} \text { 이므로 } \quad\left(\underline{\mu}_{b}-\underline{\mu}_{a}\right)^{\prime}\left(I-\Sigma_{b} \Sigma_{a}^{-1}\right)^{-1} \Sigma_{a}^{-1}\left(I-\Sigma_{b} \Sigma_{a}^{-1}\right)^{-1}\left(\underline{\mu}_{b}-\underline{\mu}_{a}\right)=0 \text {, 따라서 } \underline{\eta}_{a}=0 \text { 이 된다. 마 } \\
& \text { 찬가지로 } \underline{\eta}_{b}=0 \text { 이 된다. } \\
& \beta=\left(\underline{\mu}_{b}-\underline{\mu}_{a}\right)^{\prime}\left(\Sigma_{a}-\Sigma_{b}\right)^{-1}\left(\underline{\mu}_{b}-\underline{\mu}_{a}\right)+\left(\log \left|\Sigma_{a}\right|-\log \left|\Sigma_{b}\right|\right) \\
& \quad=\left(\log \left|\sigma_{0}^{2} I_{n}\right|-\log \left|s^{2} I_{n}\right|\right)=n \log \left(\sigma_{0}^{2} / s^{2}\right)=n \log \left(n / \chi^{2}\right) \\
& \widehat{D}_{n}(0,1)=\Phi_{n}\left(0,\left(n / \chi^{2}-1\right) I_{n}, n \log \left(n / \chi^{2}\right)\right)-\Phi_{n}\left(0,\left(1-\chi^{2} / n\right) I_{n}, n \log \left(n / \chi^{2}\right)\right)
\end{aligned}
$$

[별첨II]-2

$$
\begin{aligned}
& \Sigma_{a}^{1 / 2} \Sigma_{b}^{-1} \Sigma_{a}^{1 / 2}-I=s I_{n} \cdot \sigma_{0}^{-2} I_{n} \cdot s I_{n}-I_{n}=\left(s^{2}-\sigma_{0}^{2}\right) / \sigma_{0}^{2} \cdot I_{n}=\left(\chi^{2} / n-1\right) I_{n} \\
& I-\Sigma_{b}^{1 / 2} \Sigma_{a}^{-1} \Sigma_{b}^{1 / 2}=I-\sigma_{0} I_{n} \cdot s^{-2} I_{n} \cdot \sigma_{0} I_{n}=\left(s^{2}-\sigma_{0}^{2}\right) / s^{2} \cdot I_{n}=\left(1-n / \chi^{2}\right) I_{n} \\
& \underline{\eta}_{a}=\left(\sqrt{\left(\underline{\mu}_{b}-\underline{\mu}_{a}\right)^{\prime}\left(I-\Sigma_{b} \Sigma_{a}^{-1}\right)^{-1} \Sigma_{a}^{-1}\left(I-\Sigma_{b} \Sigma_{a}^{-1}\right)^{-1}\left(\underline{\mu}_{b}-\underline{\mu}_{a}\right)} 0 \cdots\right)^{\prime} \text { 에서 }
\end{aligned}
$$

$\underline{\mu}_{a}=\underline{\mu}_{b}$ 이므로 $\quad\left(\underline{\mu}_{b}-\underline{\mu}_{a}\right)^{\prime}\left(I-\Sigma_{b} \Sigma_{a}^{-1}\right)^{-1} \Sigma_{a}^{-1}\left(I-\Sigma_{b} \Sigma_{a}^{-1}\right)^{-1}\left(\underline{\mu}_{b}-\underline{\mu}_{a}\right)=0$, 따라서 $\eta_{a}=0$ 이 된다. 마 찬가지로 $\eta_{b}=\ell$ 이 된다. 


$$
\begin{aligned}
& \beta=\left(\mu_{b}-\underline{\mu}_{a}\right)^{\prime}\left(\Sigma_{a}-\Sigma_{b}\right)^{-1}\left(\underline{\mu}_{b}-\underline{\mu}_{a}\right)+\left(\log \left|\Sigma_{a}\right|-\log \left|\Sigma_{b}\right|\right) \\
& \quad=\left(\log \left|s^{2} I_{n}\right|-\log \left|\sigma_{0}^{2} I_{n}\right|\right)=n \log \left(s^{2} / \sigma_{0}^{2}\right)=n \log \left(\chi^{2} / n\right) \\
& \widehat{D}_{n}(0,1)=\Phi_{n}\left(0,\left(\chi^{2} / n-1\right) I_{n}, n \log \left(\chi^{2} / n\right)\right)-\Phi_{n}\left(0,\left(1-n / \chi^{2}\right) I_{n}, n \log \left(\chi^{2} / n\right)\right)
\end{aligned}
$$

\section{[별첨III]}

$$
\begin{aligned}
& \Sigma_{a}^{1 / 2} \Sigma_{b}^{-1} \Sigma_{a}^{1 / 2}-I=s_{m} I_{n} \cdot s_{p}^{-2} I_{n} \cdot s_{m} I_{n}-I_{n}=\left(s_{m}^{2}-s_{p}^{2}\right) / s_{p}^{2} \cdot I_{n}=t_{p}^{2} /(n-2) \cdot I_{n} \\
& I-\Sigma_{b}^{1 / 2} \Sigma_{a}^{-1} \Sigma_{b}^{1 / 2}=I-s_{p} I_{n} \cdot s_{m}^{-2} I_{n} \cdot s_{p} I_{n}=\left(s_{m}^{2}-s_{p}^{2}\right) / s_{m}^{2} \cdot I_{n}=\left[t_{p}^{2} /(n-2)\right] /\left[1+t_{p}^{2} /(n-2)\right] \cdot I_{n} \\
& \eta_{a}=\left(\sqrt{\left(\mu_{b}-\mu_{a}\right)^{\prime}\left(I-\Sigma_{b} \Sigma_{a}^{-1}\right)^{-1} \Sigma_{a}^{-1}\left(I-\Sigma_{b} \Sigma_{a}^{-1}\right)^{-1}\left(\mu_{b}-\mu_{a}\right)} \quad 0 \cdots 0\right)^{\prime} \text { 에서 } \\
& \left(\underline{\mu}_{b}-\underline{\mu}_{a}\right)^{\prime}\left(I-\Sigma_{b} \Sigma_{a}^{-1}\right)^{-1} \Sigma_{a}^{-1}\left(I-\Sigma_{b} \Sigma_{a}^{-1}\right)^{-1}\left(\mu_{b}-\mu_{a}\right) \\
& =\left(\bar{X}-\bar{m} I_{n}\right)^{\prime}\left(I-s_{p}^{2} I_{n} \cdot s_{m}^{-2} I_{n}\right)^{-1} \cdot s_{m}^{-2} I_{n} \cdot\left(I-s_{p}^{2} I_{n} \cdot s_{m}^{-2} I_{n}\right)^{-1} \cdot\left(\bar{x}-\bar{m} I_{n}\right) \\
& =\left\{n_{1}\left(\bar{x}_{1}-\bar{m}\right)^{2}+n_{2}\left(\bar{x}_{2}-\bar{m}\right)^{2}\right\}\left(1-s_{p}^{2} s_{m}^{-2}\right)^{-2} s_{m}^{-2}=n\left(s_{m}^{2}-s_{p}^{2}\right)\left(1-s_{p}^{2} s_{m}^{-2}\right)^{-2} s_{m}^{-2} \\
& =n\left(s_{m}^{2}-s_{p}^{2}\right) /\left[\left(1-s_{p}^{2} s_{m}^{-2}\right)^{2} s_{m}^{2}\right]=n\left[1+t_{p}^{2} /(n-2)\right] /\left[t_{p}^{2} /(n-2)\right]
\end{aligned}
$$

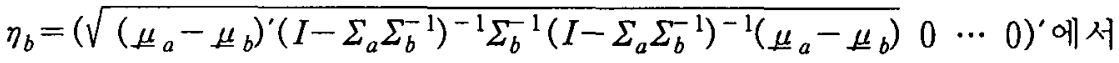

$$
\begin{aligned}
& \left(\underline{\mu}_{a}-\underline{\mu}_{b}\right)^{\prime}\left(I-\Sigma_{a} \Sigma_{b}^{-1}\right)^{-1} \Sigma_{b}^{-1}\left(I-\Sigma_{a} \Sigma_{b}^{-1}\right)^{-1}\left(\underline{\mu}_{a}-\underline{\mu}_{b}\right) \\
& =\left(\bar{m} 1_{n}-\bar{x}\right)^{\prime}\left(I-s_{m}^{2} I_{n} \cdot s_{p}^{-2} I_{n}\right)^{-1} \cdot s_{p}^{-2} I_{n} \cdot\left(I-s_{m}^{2} I_{n} \cdot s_{p}^{-2} I_{n}\right)^{-1} \cdot\left(\bar{m} 1_{n}-\bar{x}\right) \\
& =\left\{n_{1}\left(\bar{x}_{1}-\bar{m}\right)^{2}+n_{2}\left(\bar{x}_{2}-\bar{m}\right)^{2}\right\}\left(1-s_{m}^{2} s_{p}^{-2}\right)^{-2} s_{p}^{-2} \\
& =n\left(s_{m}^{2}-s_{p}^{2}\right)\left(1-s_{m}^{2} s_{p}^{-2}\right)^{-2} s_{p}^{-2}=n\left(s_{m}^{2}-s_{p}^{2}\right) /\left[\left(1-s_{m}^{2} s_{p}^{-2}\right)^{2} s_{p}^{2}\right]=n\left[t_{p}^{2} /(n-2)\right]^{-1} \\
& \beta=\left(\underline{\mu}_{b}-\underline{\mu}_{a}\right)^{\prime}\left(\Sigma_{a}-\Sigma_{b}\right)^{-1}\left(\underline{\mu}_{b}-\underline{\mu}_{a}\right)+\left(\underline{\log }\left|\Sigma_{a}\right|-\log \left|\Sigma_{b}\right|\right) \\
& =\left(\bar{x} 1_{n}-\bar{m} 1_{n}\right)^{\prime}\left(s_{m}^{2} I_{n}-s_{p}^{2} I_{n}\right)^{-1}\left(\bar{x} 1_{n}-\bar{m} 1_{n}\right)+\left(\log \left|s_{m}^{2} I_{n}\right|-\log \left|s_{p}^{2} I_{n}\right|\right) \\
& =\left[n_{1}\left(\bar{x}_{1}-m\right)^{2}+n_{2}\left(\bar{x}_{2}-m\right)^{2}\right] /\left[s_{m}^{2}-s_{p}^{2}\right]+n \log \left(s_{m}^{2} / s_{p}^{2}\right) \\
& =n+n \log \left\{1+\left(s_{m}^{2}-s_{p}^{2}\right) / s_{p}^{2}\right\}=n+n \log \left(1+t_{p}^{2} /(n-2)\right)
\end{aligned}
$$

\section{[별첨 IV]}

$$
\begin{aligned}
& \Sigma_{a}^{1 / 2} \Sigma_{b}^{-1} \Sigma_{a}^{1 / 2}-I=s_{m} I_{n} \cdot s_{p}^{-2} I_{n} \cdot s_{m} I_{n}-I_{n}=\left(s_{m}^{2}-s_{p}^{2}\right) / s_{p}^{2} \cdot I_{n}=(k-1) F /(n-k) \cdot I_{n} \\
& I-\Sigma_{b}^{1 / 2} \Sigma_{a}^{-1} \sum_{b}^{1 / 2}=I-s_{p} I_{n} s_{m}^{-2} I_{n} s_{p} I_{n}=\left(s_{m}^{2}-s_{p}^{2}\right) / s_{m}^{2} I_{n}=[(k-1) F /(n-k)] /[(k-1) F /(n-k)+1] I_{n} \\
& \eta_{a}=\left(\sqrt{\left(\underline{\mu}_{b}-\underline{\mu}_{a}\right)^{\prime}\left(I-\Sigma_{b} \Sigma_{a}^{-1}\right)^{-1} \Sigma_{a}^{-1}\left(I-\Sigma_{b} \Sigma_{a}^{-1}\right)^{-1}\left(\mu_{b}-\underline{\mu}_{a}\right)} 0 \cdots 0\right)^{\prime} \text { 에서 } \\
& \left(\underline{\mu}_{b}-\underline{\mu}_{a}\right)^{\prime}\left(I-\Sigma_{b} \Sigma_{a}^{-1}\right)^{-1} \Sigma_{a}^{-1}\left(I-\Sigma_{b} \Sigma_{a}^{-1}\right)^{-1}\left(\mu_{b}-\mu_{a}\right) \\
& =\left(\bar{x}-\bar{m} 1_{n}\right)^{\prime}\left(I-s_{p}^{2} I_{n} \cdot s_{m}^{-2} I_{n}\right)^{-1} \cdot s_{m}^{-2} I_{n} \cdot\left(I-s_{p}^{2} I_{n} \cdot s_{m}^{-2} I_{n}\right)^{-1} \cdot\left(\bar{x}-\bar{m} 1_{n}\right) \\
& =\left\{\sum_{i=1}^{k} n_{i}\left(\bar{x}_{i}-\bar{m}\right)^{2}\right\}\left(1-s_{p}^{2} s_{m}^{-2}\right)^{-2} s_{m}^{-2}=n\left(s_{m}^{2}-s_{p}^{2}\right)\left(1-s_{p}^{2} s_{m}^{-2}\right)^{-2} s_{m}^{-2}=\left[n\left(s_{m}^{2}-s_{p}^{2}\right)\right] /\left[\left(1-s_{p}^{2} s_{m}^{-2}\right)^{2} s_{m}^{2}\right] \\
& =\left[n\left(1-s_{p}^{2} / s_{m}^{2}\right)\right] /\left[\left(1-s_{p}^{2} / s_{m}^{2}\right)^{2}\right]=n /\left[\left(1-s_{p}^{2} / s_{m}^{2}\right)\right]=n\left\{1+((k-1) F /(n-k))^{-1}\right\}
\end{aligned}
$$

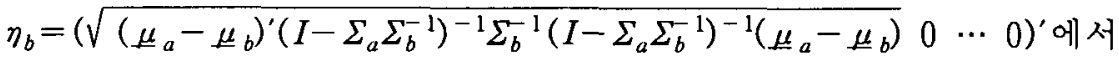

$$
\begin{aligned}
& \left(\underline{\mu}_{a}-\underline{\mu}_{b}\right)^{\prime}\left(I-\Sigma_{a} \Sigma_{b}^{-1}\right)^{-1} \Sigma_{b}^{-1}\left(I-\Sigma_{a} \Sigma_{b}^{-1}\right)^{-1}\left(\underline{\mu}_{a}-\underline{\mu}_{b}\right) \\
& =\left(\bar{m} 1_{n}-\bar{x}\right)^{\prime}\left(I-s_{m}^{2} I_{n} \cdot s_{p}^{-2} I_{n}\right)^{-1} \cdot s_{p}^{-2} I_{n} \cdot\left(I-s_{m}^{2} I_{n} \cdot s_{p}^{-2} I_{n}\right)^{-1} \cdot\left(\bar{m} 1_{n}-\bar{x}\right) \\
& =\left\{\sum_{i=1}^{k} n_{i}\left(\bar{x}_{i}-\bar{m}\right)^{2}\right\}\left(1-s_{m}^{2} s_{p}^{-2}\right)^{-2} s_{p}^{-2}=n\left(s_{m}^{2}-s_{p}^{2}\right)\left(1-s_{m}^{2} s_{p}^{-2}\right)^{-2} s_{p}^{-2} \\
& =\left[n\left(s_{m}^{2}-s_{p}^{2}\right)\right] /\left[\left(1-s_{m}^{2} s_{p}^{-2}\right)^{2} s_{p}^{2}\right]=n s_{p}^{2} /\left[\left(s_{m}^{2}-s_{p}^{2}\right)\right]=n((k-1) F /(n-k))^{-1} \\
& \beta=\left(\underline{\mu}_{b}-\underline{\mu}_{a}\right)^{\prime}\left(\Sigma_{a}-\Sigma_{b}\right)^{-1}\left(\underline{\mu}_{b}-\underline{\mu}_{a}\right)+\left(\log \left|\Sigma_{a}\right|-\log \left|\Sigma_{b}\right|\right) \\
& =\left(\bar{x} 1_{n}-\bar{m} 1_{n}\right)^{\prime}\left(s_{m}^{2} I_{n}-s_{p}^{2} I_{n}\right)^{-1}\left(\bar{x} 1_{n}-\bar{m} 1_{n}\right)+\left(\log \left|s_{m}^{2} I_{n}\right|-\log \left|s_{p}^{2} I_{n}\right|\right) \\
& =\sum_{i=1}^{k} n_{i}\left(\bar{x}_{i}-\bar{m}\right)^{2} /\left(s_{m}^{2}-s_{p}^{2}\right)+n \log \left(s_{m}^{2} / s_{p}^{2}\right)=n+n \log \{1+(k-1) F /(n-k)\}
\end{aligned}
$$

